\title{
Article
}

\section{The Art of Positronics in Contemporary Nanomaterials Science: A Case Study of Sub-Nanometer Scaled Glassy Arsenoselenides}

\author{
Oleh Shpotyuk ${ }^{1,2}$, Adam Ingram ${ }^{3}$, Catherine Boussard-Pledel ${ }^{4}$, Bruno Bureau ${ }^{4}$, Zdenka Lukáčová Bujňáková ${ }^{5}$, \\ Peter Baláž ${ }^{5}\left(\mathbb{0}\right.$, Bohdan Mahlovanyi ${ }^{4,6}$ and Yaroslav Shpotyuk ${ }^{6,7, *}$
}

1 Department of Optical Glass and Ceramics, Vlokh Institute of Physical Optics, 23 Dragomanov Str., 79005 Lviv, Ukraine; olehshpotyuk@yahoo.com

2 Faculty of Science and Technology, Jan Dlugosz University in Czestochowa, 13/15 al. Armii Krajowej, 42-200 Czestochowa, Poland

3 Department of Physics, Opole University of Technology, 75 Ozimska Str., 45-370 Opole, Poland; a.ingram@po.opole.pl

4 CNRS, ISCR, Université de Rennes, UMR 6226, F-35000 Rennes, France; catherine.boussard@univ-rennes1.fr (C.B.-P.); bruno.bureau@univ-rennes1.fr (B.B.); bmahlovanyi@ur.edu.pl (B.M.)

5 Institute of Geotechnics of Slovak Academy of Sciences, 45 Watsonova Str., 04001 Košice, Slovakia; bujnakova@saske.sk (Z.L.B.); balaz@saske.sk (P.B.)

6 Institute of Physics, University of Rzeszow, 1 Pigonia Str., 35-959 Rzeszow, Poland

7 Department of Sensor and Semiconductor Electronics, Ivan Franko National, University of Lviv, 107 Tarnavskoho Str., 79017 Lviv, Ukraine

* Correspondence: yshpotyuk@ur.edu.pl

\section{check for}

updates

Citation: Shpotyuk, O.; Ingram, A.; Boussard-Pledel, C.; Bureau, B.; Lukáčová Bujňáková, Z.; Baláž, P.; Mahlovanyi, B.; Shpotyuk, Y. The Art of Positronics in Contemporary Nanomaterials Science: A Case Study of Sub-Nanometer Scaled Glassy Arsenoselenides. Materials 2022, 15, 302. https://doi.org/10.3390/ ma15010302

Academic Editor: Mihaela Albu

Received: 23 November 2021

Accepted: 30 December 2021

Published: 1 January 2022

Publisher's Note: MDPI stays neutral with regard to jurisdictional claims in published maps and institutional affiliations.

Copyright: (c) 2022 by the authors Licensee MDPI, Basel, Switzerland This article is an open access article distributed under the terms and conditions of the Creative Commons Attribution (CC BY) license (https:// creativecommons.org/licenses/by/ $4.0 /)$.

\begin{abstract}
The possibilities surrounding positronics, a versatile noninvasive tool employing annihilating positrons to probe atomic-deficient sub-nanometric imperfections in a condensed matter, are analyzed in application to glassy arsenoselenides $\mathrm{g}-\mathrm{As}_{\mathrm{x}} \mathrm{Se}_{100-\mathrm{x}}(0<\mathrm{x}<65)$, subjected to dry and wet (in $0.5 \%$ PVP water solution) nanomilling. A preliminary analysis was performed within a modified two-state simple trapping model (STM), assuming slight contributions from bound positron-electron (Ps, positronium) states. Positron trapping in $\mathrm{g}-\mathrm{As}_{\mathrm{x}} \mathrm{Se}_{100-\mathrm{x}} / \mathrm{PVP}$ nanocomposites was modified by an enriched population of Ps-decay sites in PVP. This was proven within a three-state STM, assuming two additive inputs in an overall trapping arising from distinct positron and Ps-related states. Formalism of x3-x2-CDA (coupling decomposition algorithm), describing the conversion of Ps-decay sites into positron traps, was applied to identify volumetric nanostructurization in wet-milled g-As-Se, with respect to dry-milled ones. Under wet nanomilling, the Ps-decay sites stabilized in inter-particle triple junctions filled with PVP replaced positron traps in dry-milled substances, the latter corresponding to multi-atomic vacancies in mostly negative environments of Se atoms. With increased Se content, these traps were agglomerated due to an abundant amount of Se-Se bonds. Three-component lifetime spectra with nanostructurally- and compositionally-tuned Ps-decay inputs and average lifetimes serve as a basis to correctly understand the specific "rainbow" effects observed in the row from pelletized PVP to wet-milled, dry-milled, and unmilled samples.
\end{abstract}

Keywords: nanocomposites; mechanomilling; volumetric nanostructurization; glass; positron annihilation lifetime spectroscopy

\section{Introduction}

Chalcogenide compounds represented by glassy arsenoselenides, $\mathrm{g}-\mathrm{As}_{\mathrm{x}} \mathrm{Se}_{100-\mathrm{x}}$, from "pure" selenium g-Se to stoichiometric g-As ${ }_{40} \mathrm{Se}_{60}$ (the group of under-stoichiometric arsenoselenides) and stretched further with As content beyond arsenic triselenide $\mathrm{g}-\mathrm{As}_{2} \mathrm{Se}_{3}$ (viz. g-As ${ }_{40} \mathrm{Se}_{60}$ ), to As-rich g- $\mathrm{As}_{65} \mathrm{Se}_{35}$ (the group of over-stoichiometric arsenoselenides), compose an important family of archetypal media possessing widespread application in optoelectronics, IR photonics, opto- and space telecommunication, bio- and chemical sensing, etc. [1-3]. Nowadays, these disordered materials have also attracted interest in 
biomedicine because of their promising usage as anti-cancer drugs in the treatment of many hematological malignant diseases [4,5].

To a great extent, the medicinal usage of these compounds in contemporary therapeutics (often named arsenicals [4]) is tuned externally through nanostructurization, i.e., the technology reconstructing these materials over atomic and sub-atomic scales by influence of external stimuli, such as mechanochemistry [6-9], the high-energy mechanical milling (referred to as nanomilling, NM), ensuring unique biocompatibility in the material under treatment due to the abnormally enhanced ratio of surface-to-bulk states [10-12]. The NM is concomitant with generation of a vast variety of free-volume elements (FVE) in nanostructured material, such as vacancies, vacancy clusters and agglomerates, dislocations, some interfacial (IF) imperfections, such as inter-particle triple junctions (TJ), grain-boundaries (GB), etc., which possess an excess of atomic-deficient space as compared with non-defective close-packed conformations. In anti-microbial applications, the milled arsenicals are often combined with organic stabilizers, such as polyvinylpyrrolidone (PVP), keeping individuality of the shaped nanoparticles (NP), thus forming the PVP-capped nanocomposites $[8,9,13-15]$.

In this work, atomic-deficient structures of glassy arsenoselenides, $\mathrm{g}-\mathrm{As}_{\mathrm{x}} \mathrm{Se}_{100-\mathrm{x}}$, from glass-forming regions, covering under-stoichiometric $(0<x<40)$ and over-stoichiometric $(40<x<65)$ domains activated by NM in dry and wet mode (i.e., milled in PVP medium), was comprehensively analyzed, employing positronics, recognized as an advanced highinformative noninvasive tool probing sub-nm-scaled volumetric effects in a condensed matter, grounded on positron $\left(\mathrm{e}^{+}\right)$annihilation lifetime (PAL) spectroscopy [16-26].

\section{Materials and Methods}

\subsection{Nanocomposite Preparation and Microstructure Characterization}

The nanocomposites were prepared using coarse-grained powdered (cgp) $\mathrm{As}_{\mathrm{x}} \mathrm{Se}_{100-\mathrm{x}}$ synthesized by melt-quenching from high-purity commercial elemental precursors (As and Se of $5 \mathrm{~N}$ purity), as described in more detail elsewhere [1,2]. The cgp samples were pelletized via a conventional procedure of compressing in stainless-steel die (under $\sim 0.7 \mathrm{GPa}$ ) to produce discs ( $\sim 6 \mathrm{~mm}$ in diameter and $\sim 1 \mathrm{~mm}$ in thickness), hereafter referred to as unmilled samples.

The prepared cgp g-As $\mathrm{Se}_{100-\mathrm{x}}$ samples were nanostructurized by NM in dry and wet modes. The Pulverissete 6 (Fritsch, Idar-Oberstein, Germany) planetary ball mill operated at $500 \mathrm{rpm}$ and protective Ar atmosphere was used for dry-NM. This procedure was performed for $20 \mathrm{~min}$ in a $250 \mathrm{~mL}$ tungsten carbide chamber (loaded with 50 tungsten carbide balls, each $10 \mathrm{~mm}$ in diameter), using $3 \mathrm{~g}$ of cgp g-As $\mathrm{Se}_{100-\mathrm{x}}$ sieved under $200 \mu \mathrm{m}$. The prepared fine-grained powdered (fgp) arsenoselenides were pelletized via the same procedure as in the case of the unmilled probes.

The part of preliminary dry-milled arsenoselenides was additionally milled in a wet mode. This 90-min attrition route was performed in the presence of $300 \mathrm{~mL}$ of $0.5 \%$ PVP solution using laboratory MiniCer mill (Netzsch, Selb, Germany) operated at $3500 \mathrm{rpm}$ (85\% loading of milling shaft with yttrium-stabilized $\mathrm{ZrO}_{2}$ balls, each having $0.6 \mathrm{~mm}$ in diameter). The powdered PVP with an average molecular weight of $\mathrm{M}_{\mathrm{W}}=40,000 \mathrm{~g} \cdot \mathrm{mol}^{-1}$ purchased from Sigma-Aldrich Co. LLC (St. Louis, MO, USA) was used. The nanosuspension was dried at $70{ }^{\circ} \mathrm{C}$, and further pelletized under the same conditions, thus producing fgp wet-milled glassy arsenoselenides, representing themselves as $\mathrm{g}-\mathrm{As}_{\mathrm{x}} \mathrm{Se}_{100-\mathrm{x}} / \mathrm{PVP}$ nanocomposites.

The NP-size distribution in the nanosuspensions identified with photon cross-correlation spectroscopy using a Nanophox (Sympatec, Germany) analyzer shows single-mode dependence with $d_{50}=\sim 150-200 \mathrm{~nm}$ (i.e., 50\% of NP were smaller than 150-200 nm) and $\mathrm{d}_{99}=\sim 250-280 \mathrm{~nm}$ (i.e., $99 \%$ of NP were smaller than $250-280 \mathrm{~nm}$ ). As it follows from an X-ray diffraction analysis [8,9], the high-energy mechanical milling under the above parameters does not change the principally amorphous state of arsenicals, while essentially enhancing their degree of amorphization. 


\subsection{The PAL Spectra Recording and Preliminary Treatment}

The PAL spectra were recorded with a conventional fast-fast coincidence system (ORTEC), $230 \mathrm{ps}$ in resolution, which was stabilized at a temperature of $22^{\circ} \mathrm{C}$ and relative humidity of $35 \%$ (for more details of the PAL spectra measurements, see reference [19]). To ensure precise positron lifetimes, each spectrum was recorded in normal measuring statistics involving as high as $\sim 10^{6}$ coincidences, the channel width of $6.15 \mathrm{ps}$ being chosen to allow 8000 channels in a total per spectrum. The ${ }^{22} \mathrm{Na}$ isotope of low $\sim 50-\mathrm{kBq}$ activity prepared from aqueous ${ }^{22} \mathrm{NaCl}$ solution (wrapped by $12 \mu \mathrm{m}$ Kapton ${ }^{\circledR}$ foil and sealed) was used as the positron source sandwiched between two samples. The source correction $(12 \%$ contribution with a 0.372 ns lifetime, installed due to calibration with $\mathrm{Ni}$ and Kapton ${ }^{\circledR}$ foil) was applied to compensate the input, originating from annihilation in the source itself and Kapton ${ }^{\circledR}$ foil.

The best fitting of the collected PAL spectra was achieved with the LT 9.0 program [25] under decomposition into three constraint-free negative exponentials (so-called unconstrained x3-term fitting) obeying normalization by component intensities $\left(I_{1}+I_{2}+I_{3}=1\right)$ and stabilizing the model-independent average positron lifetime $\tau_{a v}{ }^{\Sigma}$ as the mass center of the PAL spectrum:

$$
\tau_{a v}^{\Sigma}=\sum_{i} I_{i} \tau_{i}
$$

where $\tau_{i}$ and $I_{i}$ denote positron lifetime and intensity of the respective fitting component $(i=3)$.

This approach covers different channels in substances arising from positrons annihilating in defect-free bulk (DFB) states, intrinsic trapping sites (captured in free-volume defects), and bound positron-electron $\left(\mathrm{e}^{+} \mathrm{e}^{-}\right)$states (i.e., captured in positronium Ps-decay holes) [18-20,24]. For highly inhomogeneous nanosubstances, the unconstrained x3-term fitting to the raw PAL spectra has an obvious advantage, i.e., not resulting in any artifacts related to fixing in $\tau_{i}$ lifetimes and $I_{i}$ intensities [27-29]. Under this preliminary step, the reconstructed PAL spectra allow the error-bar in lifetimes $\tau_{i}$ and intensities $I_{i}$ at the level of $\pm 0.005 \mathrm{~ns}$ and $0.5 \%$, respectively.

\subsection{Algorithmization of the Multi-Channel PAL Spectra in Nanocomposites}

In nanostructurized heterogeneous substances, such as multi-nanoparticulate composites, dominated by annihilation from both positron- and Ps-related states, the unconstrained x3-term decomposed PAL spectra can be interpreted with different mathematical algorithms based on the canonical simple trapping model (STM) [16-20]. However, in a strong physical relevance, the STM is suitable for unconstrained two-component PAL spectra in solids having only one kind of positron trap in overall positron-electron annihilation (where annihilation from Ps-related sites is negligible). Therefore, the multi-channel PAL spectra of nanosubstances having a great variety of FVE are to be processed with STM, modified for some close-to-realistic restrictions.

\subsubsection{Canonical Two-State STM Ignoring Ps-Decaying}

One of the most simplified approaches used to analyze unconstrained three-component PAL spectra in nanosubstances is canonical two-state STM [16-20] applied under conditions of slight input from the Ps-decay channel. This model describes positron (not Ps) annihilation from one kind of FVE, such as nm-sized atomic/sub-atomic voids. Being trapped by such structural defects, the positron annihilates with the $\tau_{2}=\tau_{d}$ lifetime approaching $\sim(0.2-0.5)$ ns $[18,19]$. Under a small Ps-decay contribution, the positron trapping channel is balanced by input from delocalized positrons annihilating directly from defect-free bulk states, obeying a two-state trapping scheme with a compensating $\left(\tau_{1}, I_{1}\right)$ component and average lifetime for trapped positrons $\tau_{a v}{ }^{t r}$. defined as

$$
\tau_{a v}^{t r .}=\eta_{b} \cdot \tau_{b}+\eta_{d} \cdot \tau_{d}=\tau_{1} \cdot I_{1}+\tau_{2} \cdot I_{2}
$$

where $\eta_{d}=\left(1-\eta_{b}\right)$ and $\eta_{b}$ are fractions of defect-trapped and free annihilated positrons [19]. 
Thus, within two-state STM [16-20], the shortest $\tau_{1}$ lifetime is only the reduced bulk lifetime, which is connected (but not equivalent) to the defect-free bulk positron lifetime $\tau_{b}$ :

$$
\tau_{b}=\frac{\tau_{1} \tau_{2}}{I_{1} \tau_{2}+I_{2} \tau_{1}}
$$

In this case, the positron-trapping rate in defects $\kappa_{d}$ and the fraction of trapped positrons $\eta_{d}$ can be simply calculated as

$$
\begin{gathered}
\kappa_{d}=\frac{I_{2}}{I_{1}}\left(\frac{1}{\tau_{b}}-\frac{1}{\tau_{2}}\right) \\
\eta_{d}=\frac{\kappa_{d} \cdot \tau_{b}}{1+\kappa_{d} \cdot \tau_{b}}=\tau_{1} \cdot \kappa_{d}
\end{gathered}
$$

Apart from above parameters, the difference between defect-specific and defect-free bulk lifetimes $\left(\tau_{2}-\tau_{b}\right)$ is accepted as the size signature of positron-trapping sites in terms of the equivalent number of vacancies, whereas the ratio between lifetimes $\left(\tau_{2} / \tau_{b}\right)$ is ascribed to the nature of these traps [18].

In substances affected by nanostructurization, the evolution processes related to positron trapping-responsible FVE could be identified by employing the fitting parameters and trapping modes derived from the two-state STM. Thus, the trapping rate $\kappa_{d}$ occurs to be defect dependent:

$$
\kappa_{d}=C \cdot \mu
$$

where $C$ is the defect concentration and $\mu$ is the positron trapping coefficient (which approaches $\sim 10^{15}$ atom $\cdot \mathrm{s}^{-1}$ for negative vacancies in elemental and compound semiconductors [18]).

Positron trapping-modification processes in nanostructurized substances are governed by changes in the number of positron traps, as well as in their volume and chemical environments, being principally different for small and large FVE [18-20]. A strong increase in the trapping coefficient $\mu$ is expected with the size for small vacancies, while trapping is close to saturation for large vacancies and vacancy-like clusters where the trapping rate $\kappa_{d}$ becomes strongly dependent on defect concentration $C$ [18,30]. Typical views of main modification processes in FVE, responsible for positron trapping in nanostructured substances governed by deviations in their volume and environment, are reproduced schematically in Figure 1a-d.

Under agglomeration (Figure 1a) [20,31], voids get favorable environments to grow in size due to their merging (so one can expect a decrease in the number of voids accompanied by increase in their size). In case of small voids involving no more than several vacancies (referred to as agglomeration I), a strong increase in $\tau_{d}=\tau_{2}$ (and $\mu$ ) leads to an increased trapping rate $\kappa_{d}$ despite a decrease in void concentration C. As it follows from Equation (4), these changes agree with a slight deviation in the $I_{2}$ intensity. In the case of large voids (agglomeration II), a slight increase in the $\tau_{d}=\tau_{2}$ lifetime is expected. Thus, the trapping rate $\kappa_{d}$ drops down, owing to a preferential decrease in $C$ and a slight increase in $\mu$. Consequently, the $I_{2}$ intensity decreases, as it follows from Equation (4). In an opposite case of void fragmentation, reproduced in Figure 1b [20], voids have an unfavorable environment to grow in, and tend to be tiny in size due to the dividing of a few distinct parts. For small voids (fragmentation I), one can expect a strong decrease in the $\tau_{d}=\tau_{2}$ lifetime and trapping rate $\kappa_{d}$ (with respect to a preferential decrease in the trapping coefficient $\mu$ and an increased number of voids $C$ ), resulting in slight deviations in $I_{2}$. For large voids (fragmentation II), a decrease in $\tau_{d}$ is accompanied by increased $\kappa_{d}$ (due to slightly decreased $\mu$ ), resulting from increased $I_{2}$. 
a) agglomeration

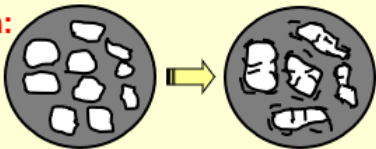

Void agglomeration I (for small voids): slightly changed $I_{2}$ and strongly increased $\tau_{2} \uparrow$ associated with

increased $\kappa_{d} \uparrow$ and $\mu \uparrow$ but decreased $C \downarrow$

Void agglomeration II (for large voids):

decreased $I_{2} \downarrow$ and slightly increased $\tau_{2} \uparrow$

associated with

decreased $\kappa_{d} \downarrow$ and $C \downarrow$ but slightly increased $\mu \uparrow$

c) expansion

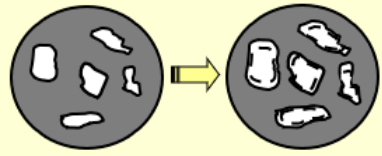

slightly decreased $I_{2} \downarrow$ and strongly increased $\tau_{2} \uparrow$ associated with

increased $\boldsymbol{\kappa}_{\boldsymbol{\phi}} \uparrow$ and $\mu \uparrow$ but unchanged $C$

e) coarsening

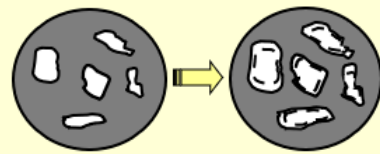

slightly increased $I_{2} \uparrow$ and $\tau_{2} \uparrow$ associated with

increased $\kappa_{d} \uparrow$ and $\mu \uparrow$ but unchanged $C$

g) charging

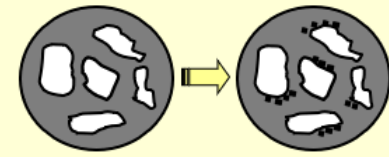

increased $I_{2}$ but slightly modified $\tau_{2}$ associated with increased $\kappa_{d} \uparrow$ and $\mu \uparrow$ b) fragmentation

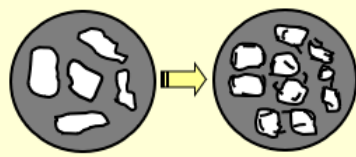

Void fragmentation I (for small voids) slightly changed $I_{2}$ and strongly decreased $\tau_{2} \downarrow$ associated with

decreased $\kappa_{o} \downarrow$ and $\mu \downarrow$ but increased $C \uparrow$

Void fragmentation II (for large voids):

increased $I_{2} \uparrow$ and slightly decreased $\tau_{2} \downarrow$

associated with

increased $\kappa_{d} \uparrow$ and $C \uparrow$ but slightly decreased $\mu \downarrow$

d) contraction

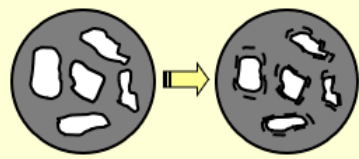

slightly increased $I_{2} \uparrow$ and strongly decreased $\tau_{2} \downarrow$ associated with

decreased $\kappa_{\alpha} \downarrow$ and $\mu \downarrow$ but unchanged $C$

f) refining

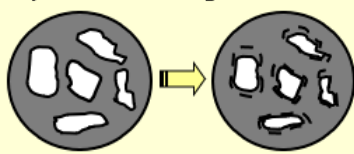

slightly decreased $I_{2} \downarrow$ and $\tau_{2} \downarrow$

associated with

decreased $\kappa_{d} \downarrow$ and $\mu \downarrow$ but unchanged $C$

h) discharging

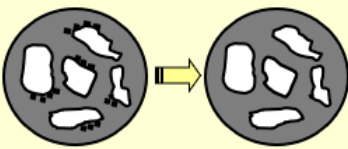

decreased $I_{2}$ but slightly modified $\tau_{2}$ associated with decreased $\kappa_{d} \downarrow$ and $\mu \downarrow$

Figure 1. Schematic cartoons showing a variety of void-modification processes associated with PALdetectable changes in positron-trapping modes ascribed to FVE under nanostructurization by preferential changes in volume ((a) - agglomeration, (b) — fragmentation, (c)—expansion, (d) — contraction) and environment ((e)—coarsening, (f) —refining, (g)—charging, (h) — discharging). Dashed counter lines correspond to sizes of positron-trapping FVE before nanostructurization.

Under void expansion, FVE have favorable environments to grow in size without essential changes in concentration $C$ [20] (Figure 1c). These changes are concomitant with increased positron trapping efficiency of voids due to excess of free volume. The expanded voids are characterized by increased $\tau_{d}=\tau_{2}$ and $\kappa_{d}$, but these increments are undetectable for large FVE, having slightly increased $\mu$. Under such conditions, an expected trend in $I_{2}$ is ambiguous, dominated by slightly decreased $I_{2}$ for nanocomposites. By analogy, void contraction (Figure 1d) [20] is considered as the process getting FVE environments, suppressing their sizes without changes in void concentration $C$, the expected changes in positron-trapping modes being opposite to those caused by void expansion.

Schematic presentation of deviation processes occurring in positron trapping-responsible FVE in nanosubstances governed by changes in void environments is shown in Figure $1 \mathrm{e}-\mathrm{h}$. Under void coarsening (Figure 1e) [20,31], FVE get favorable environments for positron trapping due to the excess of free volume at the boundary surface (also without changes in C). This process is revealed by slightly increased $\tau_{d}=\tau_{2}$ and $I_{2}$ associated with increased $\kappa_{d}$ and $\mu$. By analogy, under void refining [20], FVE get unfavorable environments for positron trapping due to reduced boundary free volume (voids get more refined, but thinner in their boundaries, see Figure 1f).

For the sake of completeness, we also consider void charging and void discharging processes, shown respectively in Figure 1g,h [20]. The void charging supposes the appearance of the positron-attractive negative charge near FVE enhancing trapping efficiency. Under void discharging, the negative charge near FVE is lost by attaining a positron- 
repulsive neutral or more positive charge. Void charging causes slightly modified $\tau_{d}=\tau_{2}$ due to changes in the annihilation rate of more negatively-charged site, but substantially increased $\kappa_{d}$ and $\mu$. In respect to Equation (4), such changes lead to increased $I_{2}$. The process of void discharging is characterized by opposite changes.

Noteworthy, strict differentiation in the above void-modification processes for nmscaled nanostructurization-driven volumetric effects is quite ambiguous in view of similarity between expected PAL-detectable responses derived, assuming unchangeable contributions in the defect-free bulk positron annihilation channel. Nevertheless, in combination with other complementary research employing conventional atomic-specific probes (such as $X$-ray diffraction analysis), the realistic nature of respective nanostructural transformations can be clarified.

Specifically, canonical two-state STM [16-20] describes positrons annihilating from two distinct sources, these being lattice-specific and defect-localized states ignoring the back escape of trapped positrons [16-20]. The remaining input in the reconstructed PAL spectra is caused by Ps-decay contribution due to positrons annihilating from Ps-related FVE (holes, voids, pores) as free particles, or interacting with electrons from the environment $[16,18,24]$. In a ground state, the Ps atom exists as para-Ps (p-Ps, antiparallel positron-electron spins) decaying intrinsically with two $\gamma$-quanta and 0.125 ns lifetime (in a vacuum), and orthoPs (o-Ps, having parallel configuration of positron-electron spins) decaying with three $\gamma$-quanta and 142 ns lifetime, these states being occupied with 1:3 probability. In a matter, since the positron wave function overlaps with the electron outside, annihilation with such electron from the environment having antiparallel spin decreases the o-Ps lifetime to $0.5-10 \mathrm{~ns}$, resulting in two $\gamma$-rays (the "pick-off" annihilation) $[18,24]$. Two conditions should be satisfied to form the Ps-decaying state in solid, the first being sufficiently great free volume available to capture the bound positron-electron system, and the second being low electron density preventing the direct annihilation [24,32]. This specificity defines selective distribution of the positron and Ps traps in nanostructured media.

The Ps atoms localized in free-volume holes of the substance gives indication on the mean radii $R$ in terms of the long-lived $\tau_{3}$ lifetime (the $I_{3}$ value correlates with the density of the Ps-decay sites), with respect to the semi-empirical Tao-Eldrup equation:

$$
\tau_{3}=0.5 \cdot\left[1-\frac{R}{R+\Delta R}+\frac{1}{2 \pi} \cdot \sin \left(\frac{2 \pi R}{R+\Delta R}\right)\right]^{-1}
$$

where $\Delta R=0.166 \mathrm{~nm}$ is the fitted empirical layer thickness [24].

By fitting the above Equation (7) with the measured longer-lived $\tau_{3}$ lifetime, the $R_{3}$ radius and respective FVE $V_{f}$ (in spherical approximation) can be determined. The fractional free volume $\left(f_{v}\right)$ in the substance under consideration where Ps-related states are stabilized can be calculated as

$$
f_{v}=C^{\prime} \cdot I_{3} \cdot V_{f}
$$

using empirical constant $C^{\prime}=0.0018 \AA^{-3}$ validated for materials without structural groups inhibiting Ps formation (such as epoxy polymers [26]).

\subsubsection{Three-State Additive (Mixed Positron-Ps) STM}

In case of stronger Ps-decaying yield, as in nanostructured substances [21-24,33-38], the collected x3-term decomposed PAL spectra reconstructed under constraint-free fitting can be analyzed in terms of the three-state positron-Ps additive STM assuming two inputs in an overall trapping process arising from trapped positrons and Ps-decaying states [39-41]. In fact, this model includes two distinct positron-trapping sources with different rates, these being $\kappa_{d 1}$ (for trapping from free positrons), and $\kappa_{d 2}$ (for trapping from Ps-decaying source):

$$
\kappa_{d 1}=I_{2}\left(\frac{1}{\tau_{1}}-\frac{1}{\tau_{2}}\right)
$$




$$
\kappa_{d 2}=I_{3}\left(\frac{1}{\tau_{1}}-\frac{1}{\tau_{3}}\right)
$$

allowing more correct estimation of the defect-free bulk positron lifetime $\tau_{B}{ }^{1-2}$ related to positron annihilation from the Bloch states [38]:

$$
\tau_{B}^{1-2}=\left(\frac{I_{1}}{\tau_{1}}+\frac{I_{2}}{\tau_{2}}+\frac{I_{3}}{\tau_{3}}\right)^{-1}
$$

Such approach is especially useful when dealing with comparative studies on different FVE in solids undergoing continuous changes in atomic-deficient structures under nanostructurization.

\subsubsection{Two-State Ps-Positron Trapping-Conversion Model (TCM)}

In substances obeying unconstrained three-term decomposed PAL spectra with essential contributions in the third channel $\left(I_{3}\right)$, which cannot be recompensated due to correction in a source contribution, two boundary cases related to annihilation from Ps-decaying sites can be roughly distinguished in terms of fitting parameters only [42]. Thus, the prevailing trend in the Ps-formation probability (decrease or increase) would result in isotypical simultaneous changes in both $I_{1}$ and $I_{3}$ intensities accompanied by an opposite change in $I_{2}$ intensity. In contrast, the changing trend in the hole density prevails, when simultaneous unison change in both $I_{2}$ and $I_{3}$ intensities are counterbalanced by an opposite change in $I_{1}$ intensity.

In highly heterogeneous materials, positron annihilation occurs through mixed positrontrapping and Ps-decaying channels, obeying the concept of selective localization of respective trapping sites [32,43]. The low-electron density holes with maximum free volume and minimum surface tension should fit to confine Ps stabilization, owing to its repulsive exchange potential, while the regions of higher electron density (negatively charged) and polarization (such as sub-nm-scaled voids) are more suitable to capture electrically charged positrons $\left(\mathrm{e}^{+}\right)$.

Therefore, for many nanosubstances, the positron-electron $\left(\mathrm{e}^{+} \mathrm{e}^{-}\right)$annihilation is expected through interconnected positron-Ps related channels, so that the Ps-decay sites are the only FVE, which can be converted under nanostructurization into positron-trapping sites (and vice versa). In this case, the formalism of generalized two-state STM modified for Ps-to-positron trapping conversion, referred to as x3-x2-CDA (coupling decomposition algorithm) [21-23], is a powerful tool used to identify nanostructurization-driven atomicdeficient volumetric effects.

The action of this algorithm is exemplified schematically in Figure 2, showing transformation of the distinct Ps-decay site (blue-colored hole), in the positron trap (green-colored void), in the substance subjected to nanostructurization (which is revealed as complete conversion of the Ps-decaying hole into the positron-trapping void). The similar trappingmodification processes can be realized due to partial occupation of free volumes ascribed to Ps-related holes by some nanostructurizing elements (the known problem of host-guest chemistry dealing with filling of open nanospaces in the host matrix by guest entities, such as atoms, molecules, atomic clusters, NP, etc. [23,44,45]). These processes reveal complete conversion from Ps-decay to positron-trapping channels in the PAL spectra, occurring at the same FVE (nominated as tailoring-nanospace problems [45]). Trapping-conversion processes are characters for polymer/filler nanocomposites (when filler NP are embedded into atomic-accessible free-volume holes of the polymeric matrix, operational as effective Ps-decay sites) $[22,23,44,45]$. Such changes can also be caused by defect formation in mechanochemistry [8-11], pressure-induced transformations, and interdiffusion processes in multi-NP systems $[45,46]$, etc. In the above cases, one deals with substances possessing unconstrained three-component PAL spectra, nanostructurized by transforming initial Ps-decaying holes into free positron self-annihilation sub-nm-scaled voids without contributions from other competitive channels. Within this approach, the straightforward 
parameterization of nanostructurization-driven volumetric effects can be developed using the modified STM, describing the transition from the three-component to the generalized two-component PAL spectra.
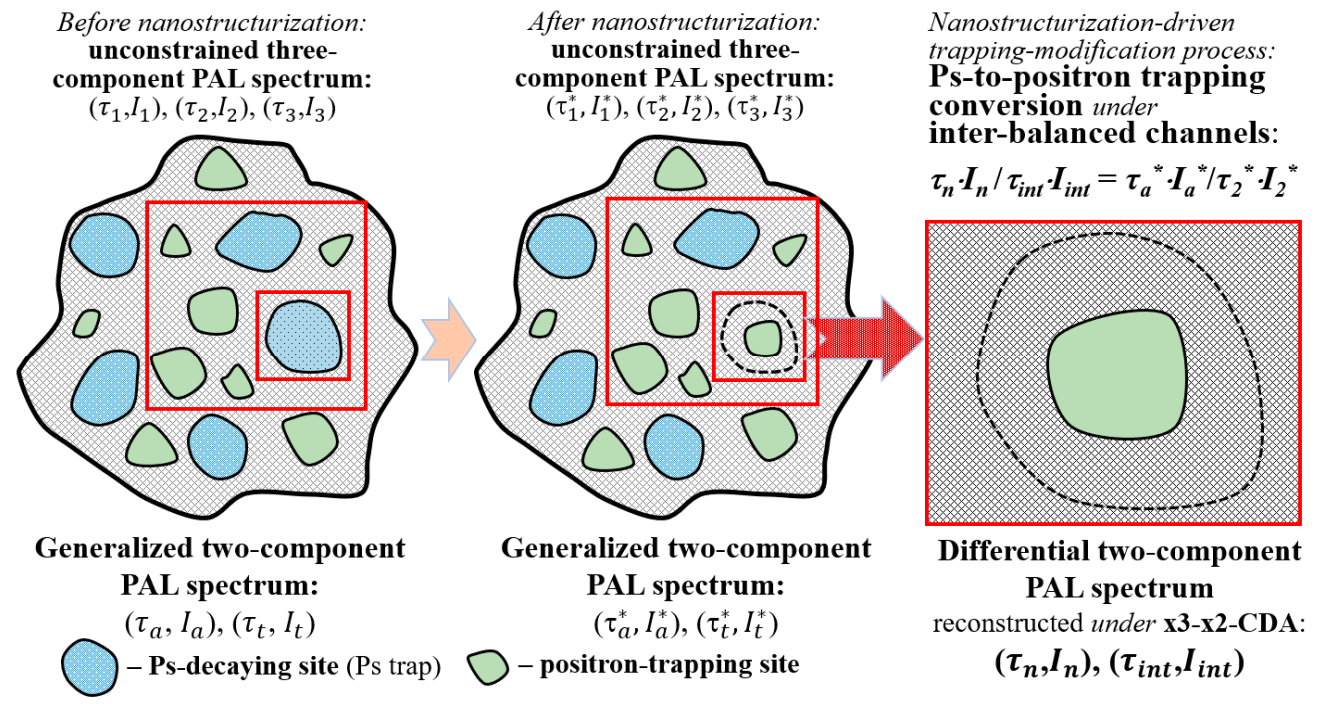

Figure 2. Fragment of atomic-deficient microstructure of substance under nanostructurization obeying completely inter-balanced Ps-to-positron trapping conversion in terms of $\mathrm{x} 3-\mathrm{x} 2-\mathrm{CDA}$. Nanostructurization is revealed as the transformation of the Ps-decaying hole (blue-color, shown by the counter-dashed line after nanostructurization) into the positron-trapping site (green-color).

Firstly, this analysis is to be applied to the unstructured matrix having $\left(\tau_{1}, I_{1}\right),\left(\tau_{2}, I_{2}\right)$, $\left(\tau_{3}, I_{3}\right)$ inputs in unconstrained $x 3$-term decomposed PAL spectrum obeying normalization $I_{1}+I_{2}+I_{3}=1$ (see Figure 2).

This three-component PAL spectrum can be transferred to the generalized twocomponent form by removing inputs from $\mathrm{p}$-Ps annihilation (with $\tau_{\mathrm{p}}=0.125 \mathrm{~ns}$ lifetime and $I_{\mathrm{p}}=I_{3} / 3$ intensity) from the first channel and o-Ps "pick-up" annihilation (with $\tau_{3}$ lifetime and $I_{3}$ intensity) from the third channel to the general trapping channel. Thus, we can simply estimate contribution $\left(\tau_{a}, I_{a}\right)$ to the first channel other than p-Ps:

$$
\begin{gathered}
\tau_{a} \cdot I_{a}=\tau_{1} \cdot I_{1}-\tau_{p} \cdot I_{p} \\
I_{a}=I_{1}-I_{p}
\end{gathered}
$$

The similar calculations should be performed for nanostructurized substance having $\left(\tau_{1}{ }^{*}, I_{1}{ }^{*}\right),\left(\tau_{2}{ }^{*}, I_{2}{ }^{*}\right),\left(\tau_{3}{ }^{*}, I_{3}{ }^{*}\right)$ inputs in the unconstrained three-component PAL spectrum (normalized by intensities $I_{1}{ }^{*}+I_{2}{ }^{*}+I_{3}{ }^{*}=1$ ), assuming the second component in the generalized PAL spectrum, as in the initial unstructured matrix (see Figure 3):

$$
\begin{gathered}
I_{2}{ }^{*}=I_{\text {int }}+I_{3}{ }^{*} \cdot\left(I_{2} / I_{3}\right) \\
\tau_{2}{ }^{*} \cdot I_{2}{ }^{*}=\tau_{\text {int }} \cdot I_{\text {int }}+\tau_{2} \cdot\left(I_{2}{ }^{*}-I_{\text {int }}\right)
\end{gathered}
$$

Nanostructurization-driven Ps-to-positron trapping conversion can be refined by the transition to the differential two-component PAL spectrum with first and second component inputs defined as

$$
\begin{gathered}
\tau_{n} \cdot I_{n}=\tau_{a}{ }^{*} \cdot I_{a}{ }^{*}-\tau_{a} \cdot\left(I_{a}{ }^{*}-I_{n}\right) \\
\tau_{\text {int }} \cdot I_{\text {int }}=\tau_{2}{ }^{*} \cdot I_{2}{ }^{*}-\tau_{2} \cdot\left(I_{2}{ }^{*}-I_{\text {int }}\right)
\end{gathered}
$$


assuming full inter-channel balance in the substance under nanostructurization (the contributions from derived components are equilibrated with those from which these components arise [21]):

$$
\tau_{n} \cdot I_{n} / \tau_{\text {int }} \cdot I_{\text {int }}=\tau_{a}^{*} \cdot I_{a}^{*} / \tau_{2}^{*} \cdot I_{2}{ }^{*}
$$

x3-term decomposed PAL spectra for initial and nanostructurized probes

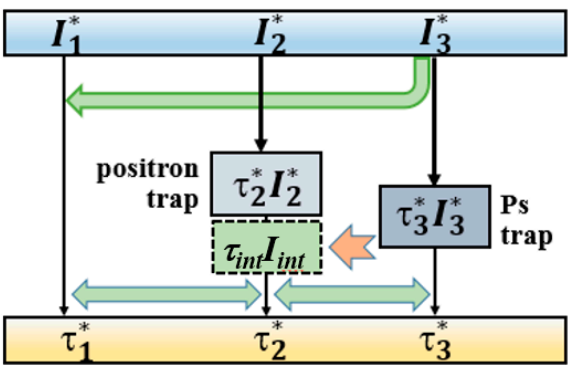

First step:

three-component PAL spectra recording and preliminary treatment (unconstrained $\mathrm{x} 3$-term fitting)

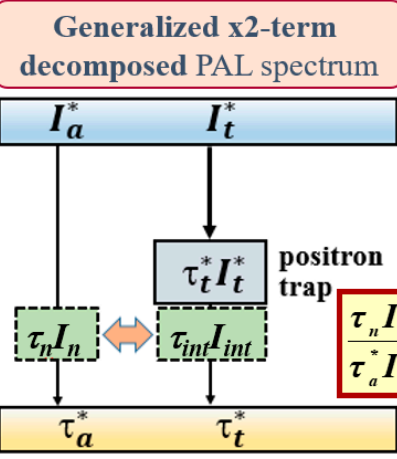

Second step:

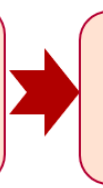

transition to generalized two-component PAL spectra (separated positron-Ps inputs)
Differential x2-term decomposed PAL spectrum

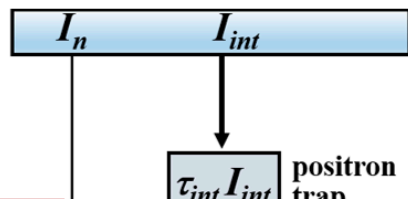

$\tau_{\text {int }} I_{\text {int }}$ trap

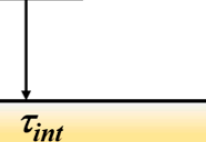

Third step:

transition to differential two-component PAL spectrum (nanostructured traps)

Figure 3. Schematic presentation of algorithm parameterizing differential two-component PAL spectrum of nanosubstance obeying Ps-to-positron trapping conversion, in terms of x3-x2-CDA. The unconstrained three-component PAL spectra of initial and nanostructurized substances are first collected, and transformed to the generalized two-component form, assuming completely inter-balanced positron-Ps contributions. Differential two-component PAL spectrum with "pure" nanostructurization-related contributions $\left(\tau_{n}, I_{n}\right)$ and $\left(\tau_{i n t}, I_{\text {int }}\right)$ is reconstructed.

Complete parameterization of Ps-to-positron trapping conversion in the nanostructurized matrix (as shown in Figures 2 and 3 ) is performed, accepting $\left(\tau_{n}, I_{n}\right)$ and $\left(\tau_{\text {int }}, I_{\text {int }}\right)$ as the respective first and second components of the reconstructed differential PAL spectrum, employing formalism of conventional two-state STM [16-20].

Within this approach, the preferential directionality in the trapping conversion is defined by the sign of both $\left(I_{n}\right.$ and $\left.I_{\text {int }}\right)$ intensities (if both intensities are positive, the direct Ps-to-positron trapping conversion occurs; while if both intensities are negative, the inverse positron-to-Ps trapping conversion occurs) [21-23]. Trapping modes derived from the Ps-positron trapping-conversion model in accord to the above Equations (2)-(5) are signatures of the nanostructured (NP-modified) matrix, involving both Ps-decay and positron-trapping related sites, these being defect-specific $\tau_{\text {int }}$ and defect-free bulk $\tau_{b}{ }^{N P}$ lifetimes, trapping rate in defects $\kappa_{d} N P$, and some derivative characteristics, relevant to the trap size, in terms of the equivalent number of vacancies defined by $\left(\tau_{\text {int }}-\tau_{b}{ }^{N P}\right)$ difference and nature of these traps, defined by $\tau_{\text {int }} / \tau_{b}{ }^{N P}$ ratio.

In contrast to the free-constraint $x 3$-term decomposition parameterizing the PAL spectra, in terms of simple uncorrelated positron-Ps trapping, the $x 3-x 2-C D A$ allows identification of separated contributions in the nanostructurized substance. However, this approach becomes meaningless, if competitive contributions from other trapping-modification processes are possible under nanostructurization (e.g., changes in annihilation from delocalized Bloch states, or changes in the population of other FVE responsible for positron and/or Ps-trapping). Furthermore, this approach is inefficient, if both $\left(\tau_{n}, I_{n}\right)$ and $\left(\tau_{\text {int }}, I_{\text {int }}\right)$ components are unsaturated under nanostructurization, allowing only negligible contributions in the overall balance of annihilation events. Thereby, the x3-x2-CDA can be used as indicative, separating processes of nanostructurization-driven host matrix modification from the simple interplay between distinct uncorrelated positron-trapping and Ps-decaying channels. 


\section{Results and Discussion}

In the current research, the collected PAL spectra of pelletized arsenoselenides $\mathrm{As}_{\mathrm{x}} \mathrm{Se}_{100-\mathrm{x}}$ were adequately reconstructed with an unconstrained $x 3$-term decomposition routine. As an example, three-component PAL spectra of cgp (unmilled) and fgp (dry-milled and wet-milled) pellets of $\mathrm{g}-\mathrm{As}_{2} \mathrm{Se}_{3}$ are reproduced at the general background of the source contribution in Figure 4.
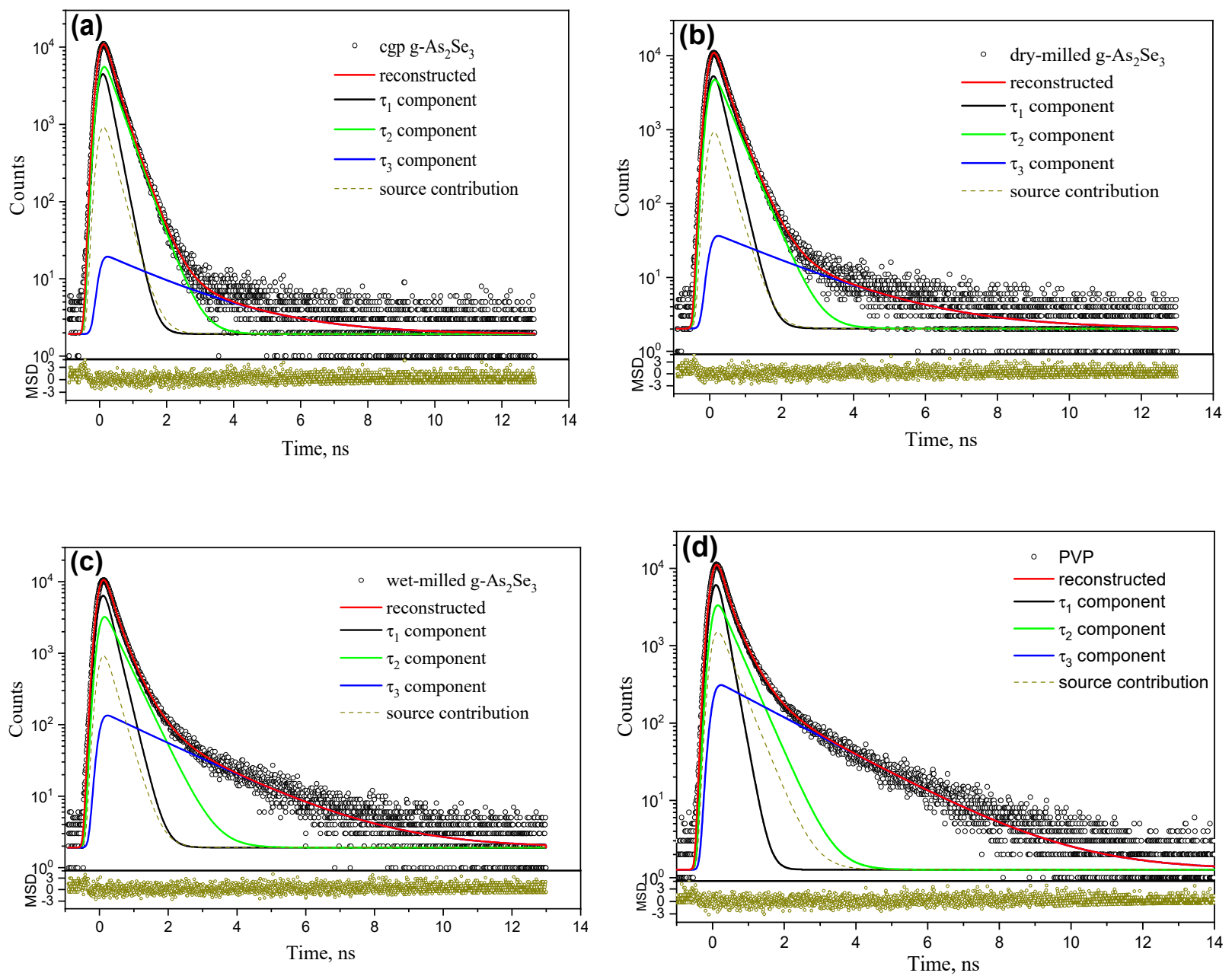

Figure 4. The PAL spectra of pelletized unmilled (a), dry-milled (b), and wet-milled $\mathrm{g}-\mathrm{As}_{2} \mathrm{Se}_{3}$ (c) reconstructed from unconstrained x3-term fitting at the general background of the source contribution. The PAL spectrum of PVP pellets reconstructed under the same decomposition using $2 \mathrm{M}$ of accumulated annihilation events [47] is shown for comparison (d). The bottom inset represents the statistical scatter of variance in favor of good applicability of the applied fitting.

Good applicability of this fitting with three unconstrained components is proved by the statistical scatter of variance (minimal statistically weighted least-square deviation between the experimental and theoretical curve built of three exponentials) tightly grouped along the time axis. For comparison, the PAL spectrum of "pure" PVP pellets reconstructed under the same routine using $2 \mathrm{M}$ of annihilation events taken from our preliminary research [47] is also reproduced in Figure 4d. The respective PAL spectra fitting parameters for pelletized g-As $\mathrm{Se}_{100-\mathrm{x}}$ and PVP are presented in Table 1. 
Table 1. Fitting parameters describing unconstrained $x 3$-term decomposed PAL spectra of pelletized unmilled, dry-milled and wet-milled g-As $\mathrm{Se}_{100-\mathrm{x}}$ (fitting parameters for PVP pellets [47] are included for comparison).

\begin{tabular}{|c|c|c|c|c|c|c|c|}
\hline \multirow{3}{*}{$\begin{array}{c}\text { Samples } \\
\text { g-As } \mathrm{Se}_{100-\mathrm{x}}, \mathrm{x}\end{array}$} & \multirow{3}{*}{$\chi=[$ FIT -1$]$} & \multicolumn{5}{|c|}{ PAL Spectra Fitting Parameters } & \multirow{2}{*}{$\tau_{a v}$} \\
\hline & & $\tau_{1}$ & $\tau_{2}$ & $\tau_{3}$ & $I_{2}$ & $I_{3}$ & \\
\hline & & ns & ns & ns & a.u. & a.u. & ns \\
\hline PVP pellets [47] & 0.20 & 0.196 & 0.472 & 1.867 & 0.256 & 0.119 & 0.466 \\
\hline Unmilled, $x=5$ & 0.04 & 0.217 & 0.377 & 2.000 & 0.300 & 0.011 & 0.285 \\
\hline Dry-milled, $x=5$ & 0.04 & 0.210 & 0.369 & 2.137 & 0.340 & 0.011 & 0.285 \\
\hline Wet-milled, $x=5$ & 0.03 & 0.212 & 0.495 & 1.764 & 0.303 & 0.112 & 0.472 \\
\hline Unmilled, $x=10$ & 0.03 & 0.216 & 0.380 & 2.232 & 0.290 & 0.012 & 0.288 \\
\hline Dry-milled, $x=10$ & 0.04 & 0.212 & 0.360 & 2.112 & 0.350 & 0.011 & 0.285 \\
\hline Wet-milled, $x=10$ & 0.07 & 0.202 & 0.438 & 1.708 & 0.348 & 0.101 & 0.436 \\
\hline Unmilled, $x=20$ & 0.03 & 0.213 & 0.368 & 2.103 & 0.340 & 0.015 & 0.294 \\
\hline Dry-milled, $x=20$ & 0.04 & 0.215 & 0.368 & 2.157 & 0.330 & 0.011 & 0.287 \\
\hline Wet-milled, $x=20$ & 0.07 & 0.211 & 0.454 & 1.706 & 0.303 & 0.086 & 0.413 \\
\hline Unmilled, $x=29$ & 0.03 & 0.212 & 0.365 & 2.117 & 0.370 & 0.012 & 0.291 \\
\hline Dry-milled, $x=29$ & 0.05 & 0.212 & 0.368 & 2.057 & 0.360 & 0.011 & 0.290 \\
\hline Wet-milled, $x=29$ & 0.19 & 0.206 & 0.390 & 1.747 & 0.342 & 0.042 & 0.334 \\
\hline Unmilled, $x=40$ & 0.01 & 0.193 & 0.358 & 2.091 & 0.620 & 0.007 & 0.309 \\
\hline Dry-milled, $x=40$ & 0.05 & 0.202 & 0.371 & 2.087 & 0.540 & 0.015 & 0.322 \\
\hline Wet-milled, $x=40$ & 0.07 & 0.212 & 0.428 & 1.891 & 0.400 & 0.052 & 0.386 \\
\hline Unmilled, $x=45$ & 0.04 & 0.185 & 0.352 & 1.964 & 0.670 & 0.008 & 0.311 \\
\hline Dry-milled, $x=45$ & 0.06 & 0.204 & 0.379 & 2.337 & 0.520 & 0.015 & 0.327 \\
\hline Wet-milled, $x=45$ & 0.08 & 0.201 & 0.405 & 1.855 & 0.500 & 0.047 & 0.382 \\
\hline Unmilled, $x=50$ & 0.06 & 0.205 & 0.370 & 2.106 & 0.540 & 0.015 & 0.323 \\
\hline Dry-milled, $x=50$ & 0.05 & 0.210 & 0.378 & 2.264 & 0.520 & 0.013 & 0.324 \\
\hline Wet-milled, $x=50$ & 0.08 & 0.207 & 0.411 & 1.833 & 0.440 & 0.049 & 0.381 \\
\hline Unmilled, $x=55$ & 0.07 & 0.207 & 0.370 & 2.183 & 0.510 & 0.014 & 0.318 \\
\hline Dry-milled, $x=55$ & 0.06 & 0.195 & 0.369 & 2.128 & 0.570 & 0.015 & 0.323 \\
\hline Wet-milled, $x=55$ & 0.08 & 0.202 & 0.419 & 1.845 & 0.460 & 0.065 & 0.409 \\
\hline Unmilled, $x=60$ & 0.05 & 0.194 & 0.359 & 2.108 & 0.580 & 0.015 & 0.318 \\
\hline Dry-milled, $x=60$ & 0.05 & 0.204 & 0.382 & 2.339 & 0.520 & 0.015 & 0.329 \\
\hline Wet-milled, $x=60$ & 0.06 & 0.216 & 0.442 & 1.903 & 0.400 & 0.059 & 0.406 \\
\hline Unmilled, $x=65$ & 0.06 & 0.207 & 0.367 & 2.098 & 0.520 & 0.015 & 0.319 \\
\hline Dry-milled, $x=65$ & 0.04 & 0.198 & 0.371 & 2.180 & 0.560 & 0.015 & 0.333 \\
\hline Wet-milled, $x=65$ & 0.07 & 0.202 & 0.403 & 1.914 & 0.470 & 0.049 & 0.380 \\
\hline
\end{tabular}

\subsection{Volumetric Nanostructurization in $g-A s_{x} S e_{100-x}$ under Modified Two-State STM}

Within a conventional case involving mixed positron-Ps annihilation channels, an adequate resolution exists only for some close-to-boundary restrictions ignoring either Ps-decaying or positron-trapping contributions. The latter are more realistic, at least, for cgp and fgp dry-milled pellets having a low third component $I_{3}$ intensity (Table 1 ).

The trapping modes derived from unconstrained $x 3$-term decomposed PAL spectra of pelletized unmilled, dry-milled, and wet-milled g- $\mathrm{As}_{\mathrm{x}} \mathrm{Se}_{100-\mathrm{x}}$, assuming two-state STM 
with ignored Ps-decay contribution are given in Table 2 (for comparison, the data for PVP pellets derived under the same decomposition route [47] are also included).

Table 2. Trapping modes derived from unconstrained $x 3$-term decomposed PAL spectra of pelletized unmilled, dry-milled, and wet-milled g- $\mathrm{As}_{\mathrm{x}} \mathrm{Se}_{100-\mathrm{x}}$, assuming two-state STM with ignored Ps-decay contribution (trapping modes for PVP pellets [47] are included for comparison).

\begin{tabular}{|c|c|c|c|c|c|c|c|c|}
\hline \multirow{3}{*}{$\begin{array}{c}\text { Samples } \\
\text { g-As }_{\mathbf{x}} \mathrm{Se}_{100-\mathrm{x}}, \mathrm{x}\end{array}$} & \multicolumn{6}{|c|}{ Positron Trapping Modes } & \multicolumn{2}{|c|}{ Ps-Decay Modes } \\
\hline & $\tau_{a v}{ }^{t r}$. & $\tau_{b}$ & $\kappa_{d}$ & $\tau_{2}-\tau_{b}$ & $\tau_{2} / \tau_{b}$ & $\eta_{d}$ & $R_{3}$ & $f_{v}$ \\
\hline & ns & ns & $\mathrm{ns}^{-1}$ & ns & a.u. & a.u. & nm & $\%$ \\
\hline PVP pellets [47] & 0.276 & 0.236 & 0.87 & 0.24 & 1.99 & 0.17 & 0.276 & 1.88 \\
\hline Unmilled, $x=5$ & 0.276 & 0.249 & 0.59 & 0.13 & 1.52 & 0.13 & 0.288 & 0.20 \\
\hline Dry-milled, $x=5$ & 0.265 & 0.247 & 0.71 & 0.12 & 1.50 & 0.15 & 0.300 & 0.23 \\
\hline Wet-milled, $x=5$ & 0.309 & 0.263 & 0.92 & 0.23 & 1.88 & 0.20 & 0.271 & 1.60 \\
\hline Unmilled, $x=10$ & 0.264 & 0.247 & 0.59 & 0.13 & 1.54 & 0.13 & 0.307 & 0.26 \\
\hline Dry-milled, $x=10$ & 0.264 & 0.248 & 0.69 & 0.11 & 1.45 & 0.15 & 0.298 & 0.21 \\
\hline Wet-milled, $x=10$ & 0.293 & 0.255 & 1.03 & 0.18 & 1.72 & 0.21 & 0.259 & 1.32 \\
\hline Unmilled, $x=20$ & 0.267 & 0.249 & 0.68 & 0.12 & 1.48 & 0.15 & 0.297 & 0.29 \\
\hline Dry-milled, $x=20$ & 0.266 & 0.250 & 0.65 & 0.12 & 1.47 & 0.14 & 0.301 & 0.22 \\
\hline Wet-milled, $x=20$ & 0.292 & 0.257 & 0.84 & 0.20 & 1.77 & 0.18 & 0.259 & 1.13 \\
\hline Unmilled, $x=29$ & 0.269 & 0.251 & 0.74 & 0.11 & 1.45 & 0.16 & 0.298 & 0.24 \\
\hline Dry-milled, $x=29$ & 0.271 & 0.253 & 0.70 & 0.12 & 1.47 & 0.15 & 0.293 & 0.21 \\
\hline Wet-milled, $x=29$ & 0.272 & 0.248 & 0.82 & 0.14 & 1.58 & 0.17 & 0.263 & 0.57 \\
\hline Unmilled, $x=40$ & 0.296 & 0.271 & 1.49 & 0.09 & 1.32 & 0.29 & 0.296 & 0.15 \\
\hline Dry-milled, $x=40$ & 0.295 & 0.269 & 1.24 & 0.10 & 1.37 & 0.25 & 0.296 & 0.29 \\
\hline Wet-milled, $x=40$ & 0.303 & 0.269 & 1.00 & 0.16 & 1.58 & 0.21 & 0.277 & 0.83 \\
\hline Unmilled, $x=45$ & 0.298 & 0.272 & 1.73 & 0.08 & 1.30 & 0.32 & 0.270 & 0.11 \\
\hline Dry-milled, $x=45$ & 0.296 & 0.270 & 1.20 & 0.11 & 1.40 & 0.24 & 0.318 & 0.35 \\
\hline Wet-milled, $x=45$ & 0.308 & 0.273 & 1.31 & 0.14 & 1.54 & 0.26 & 0.274 & 0.72 \\
\hline Unmilled, $x=50$ & 0.295 & 0.271 & 1.19 & 0.10 & 1.36 & 0.24 & 0.298 & 0.29 \\
\hline Dry-milled, $x=50$ & 0.299 & 0.274 & 1.12 & 0.10 & 1.38 & 0.23 & 0.311 & 0.30 \\
\hline Wet-milled, $x=50$ & 0.301 & 0.268 & 1.10 & 0.14 & 1.53 & 0.23 & 0.272 & 0.74 \\
\hline Unmilled, $x=55$ & 0.291 & 0.268 & 1.10 & 0.10 & 1.38 & 0.23 & 0.304 & 0.30 \\
\hline Dry-milled, $x=55$ & 0.296 & 0.268 & 1.40 & 0.10 & 1.38 & 0.27 & 0.300 & 0.31 \\
\hline Wet-milled, $x=55$ & 0.309 & 0.271 & 1.26 & 0.15 & 1.25 & 0.26 & 0.273 & 0.99 \\
\hline Unmilled, $x=60$ & 0.291 & 0.266 & 1.40 & 0.09 & 1.35 & 0.27 & 0.298 & 0.30 \\
\hline Dry-milled, $x=60$ & 0.298 & 0.271 & 1.21 & 0.11 & 1.41 & 0.25 & 0.318 & 0.36 \\
\hline Wet-milled, $x=60$ & 0.312 & 0.276 & 1.01 & 0.17 & 1.61 & 0.22 & 0.278 & 0.97 \\
\hline Unmilled, $x=65$ & 0.291 & 0.269 & 1.11 & 0.10 & 1.37 & 0.23 & 0.296 & 0.30 \\
\hline Dry-milled, $x=65$ & 0.293 & 0.266 & 1.29 & 0.10 & 1.35 & 0.26 & 0.304 & 0.32 \\
\hline Wet-milled, $x=65$ & 0.301 & 0.268 & 1.22 & 0.14 & 1.22 & 0.25 & 0.279 & 0.80 \\
\hline
\end{tabular}

Three-component spectrum of pelletized unmilled (cgp) arsenic triselenide g- $\mathrm{As}_{2} \mathrm{Se}_{3}$ reveals a second component with a defect-specific positron lifetime $\tau_{2} \sim 0.358 \mathrm{~ns}$ (see Table 1), which is at the character level for triple/quadruple atomic vacancies in binary gAs-Se [48-53]. The third component with relatively small fractional free volume $f_{v}=0.15 \%$ 
and long-lived lifetime $\tau_{3} \sim 2.091 \mathrm{~ns}$ can be ascribed to Ps-related holes with radius approaching $R \sim 0.296 \mathrm{~nm}$. Despite a small concentration of these holes (since $I_{3}$ intensity is less than $1 \%$ ), this component cannot be eliminated from consideration without substantial reduction in the fitting goodness. Nevertheless, we also decomposed PAL spectrum in (evidently worse) two distinct components, to compare these data with those for the same bulk $\mathrm{g}-\mathrm{As}_{2} \mathrm{Se}_{3}$ treated within two-state STM [50]. The revealed excellent coincidence in defect-specific $\tau_{d}=\tau_{2}$ and defect-free $\tau_{b}$ positron lifetimes, as well as trapping rate in defects $\kappa_{d}$, speaks in a favor of tight proximity between these samples.

The most simplified insight on nanostructurization-driven volumetric changes in $\mathrm{g}$-As-Se can be done in terms of the average positron lifetime $\tau_{a v}$. (1) derived from canonical STM.

In the batch of pelletized unmilled $\mathbf{g}-\mathbf{A s}_{\mathbf{x}} \mathbf{S e}_{\mathbf{1 0 0}-\mathbf{x}}$, with deviation from stoichiometry $\mathrm{As}_{2} \mathrm{Se}_{3}$ towards Se-rich compositions, the $\tau_{a v}$. lifetime derived from three-component PAL spectra $(0.309 \mathrm{~ns})$ decreases to $\sim 0.285 \mathrm{~ns}$, while demonstrating slow increase to $\sim 0.32 \mathrm{~ns}$ with approaching As-rich samples (see Table 1). The similar behavior (albeit without detectable changes for As-rich probes) is character for $\tau_{a v}{ }^{t r}$. lifetime (2), calculated for trapped positrons within STM, ignoring Ps-decay contribution (see Table 2). This compositional trend (excepting over-stoichiometric arsenoselenides) is concomitant with a sharp increase in the $\tau_{a v}$. lifetime for bulk g-As-Se obtained, by quenching from a melt approaching g- $\mathrm{As}_{2} \mathrm{Se}_{3}$ [54], where trapping occurs in intrinsic sub-nm-scaled FVE, such as agglomerates of bond-free solid angles allocated at Se atoms in the bottom of the interlinked AsSe $3 / 2$ pyramidal units [49-51,55]. More moderated behavior of $\tau_{a v}$. in these glasses above $\mathrm{As}_{2} \mathrm{Se}_{3}$ composition is caused by stabilization of molecular-network conformations dominated by realgar-type $\mathrm{As}_{4} \mathrm{Se}_{4}$ units [56]. From this analysis, it is clear that additional positron trapping sites are generated in over-stoichiometric $\mathrm{g}-\mathrm{As}_{\mathrm{x}} \mathrm{Se}_{100-\mathrm{x}}(\mathrm{x}>40)$ under coarse powdering, followed by the pelletization procedure.

In the batch of pelletized fgp dry-milled $\mathbf{g}-\mathbf{A s}_{\mathbf{x}} \mathbf{S e}_{\mathbf{1 0 0}-\mathbf{x}}$, compositional dependence of both average lifetimes $\tau_{a v}$. (1) and $\tau_{a v}{ }^{t r}$. (2) do not change substantially (see Tables 1 and 2), but show somewhat lower values for under-stoichiometric samples $(x<40)$, and higher values for over-stoichiometric samples $(x \geq 40)$. This specificity is connected with a recently discovered enhanced milling effect on molecular confirmations in this system $[57,58]$.

Neglecting o-Ps-decaying contributions in the reconstructed PAL spectra (believed to be quite reasonable for both unmilled and dry-milled $\mathrm{g}-\mathrm{As}_{\mathrm{x}} \mathrm{Se}_{100-\mathrm{x}}$ in view of $I_{3}<1.5 \%$ ), it is clear that slight agglomeration of large positron traps (the process of void agglomeration II depicted in Figure 1a) is expected in transition from unmilled to dry-milled arsenoselenides. Indeed, under this transition, a decrease in $I_{2}$ intensity is accompanied by a slight increase in $\tau_{2}$ lifetime (Table 1 ), resulting in a merely depressed positron trapping rate in defects $\kappa_{d}$ (Table 2). The only exception can be found at the edges of the glass-forming region in g-As $\mathrm{Se}_{100-\mathrm{x}}$ system (for $\mathrm{x}=5,10,55,65$ ).

The most prominent changes are found in the batch of pelletized fgp wet-milled $\mathbf{g}-\mathrm{As}_{\mathbf{x}} \mathrm{Se}_{\mathbf{1 0 0 - x}}$. In these $\mathrm{g}-\mathrm{As}_{\mathrm{x}} \mathrm{Se}_{100-\mathrm{x}} / \mathrm{PVP}$ nanocomposites, the average lifetimes for trapped positrons $\tau_{a v}{ }^{t r}$. are slightly enriched on $\sim 0.02-0.03 \mathrm{~ns}$ in respect to the same values in unmilled samples (Table 2), while average lifetimes derived from three-component PAL spectra $\tau_{a v}$. (1) demonstrate a sharp 0.07-0.08 ns increment for over-stoichiometric As-rich probes, and $\sim 0.15-0.19 \mathrm{~ns}$ increment for under-stoichiometric Se-rich probes (Table 1). Thus, in $\mathrm{g}-\mathrm{As}_{60} \mathrm{Se}_{40} / \mathrm{PVP}$ and $\mathrm{g}-\mathrm{As}_{5} \mathrm{Se}_{95} / \mathrm{PVP}$ nanocomposites, the $\tau_{a v}$. approaches $0.466 \mathrm{~ns}$ and $0.472 \mathrm{~ns}$, the values far beyond $\tau_{a v} . \sim 0.386 \mathrm{~ns}$ detected for stoichiometric $\mathrm{g}-\mathrm{As}_{40} \mathrm{Se}_{60} / \mathrm{PVP}$. As a result, compositional dependence of $\tau_{a v}$. is changed drastically, attaining broad minimum near PVP-capped $\mathrm{g}-\mathrm{As}_{2} \mathrm{Se}_{3}$. Of course, because of a strong contribution from o-Ps decaying in the reconstructed PAL spectra (Figure 4c), their analysis, in terms of alone positron trapping modes [20], is impossible.

It is worth mentioning, in the studied $\mathrm{As}_{\mathrm{x}} \mathrm{Se}_{100-\mathrm{x}} / \mathrm{PVP}$ nanocomposites, the longest lifetime $\tau_{3}$ is dropped to $\sim 1.7-1.9 \mathrm{~ns}$, tending to o-Ps-related lifetime in "pure" PVP $\left(\tau_{3}=1.867 \mathrm{~ns}\right)$ [47]. This means that Ps atoms are stabilized rather in the PVP environment 
than in g-As-Se matrix, their decay being substantially enhanced in the fractional free volumes $f_{v}$ (see Table 2 ).

Thereby, in comparison with unmilled (cgp) and dry-milled glassy arsenoselenides, positron trapping in PVP-capped g-As $\mathrm{As}_{\mathrm{x}} \mathrm{S}_{100-\mathrm{x}}$ (wet-milled probes) is modified because of enriched Ps-decaying states stabilized in a preferentially "pure" PVP environment.

In inhomogeneous multi-particulate substances composed of agglomerated NP, the large portion of positrons thermalized inside grains travel towards GB and interparticle $\mathrm{TJ}$ (since their diffusion lengths become comparable with grain size), and annihilate after getting trapped into these trapping sites. Because of a large amount of FVE generated at NM [59-61], this specificity determines the complication in the PAL spectra collected for fgp (dry-milled and wet-milled) samples as compared with cgp (unmilled) samples.

The PAL spectra of pelletized dry-milled g- $\mathrm{As}_{2} \mathrm{Se}_{3}$ demonstrates increased $\tau_{2} \sim 0.371 \mathrm{~ns}$ as compared with this value in the unmilled probe, but rather unchanged Ps-related $\tau_{3} \sim 2.087 \mathrm{~ns}$ lifetime (Table 1 ). Meanwhile, the average positron lifetime $\tau_{a v}$ is only slightly increased in this sample under NM, the similar tendencies being observed in other arsenoselenides with average deviation of $\pm 0.01 \mathrm{~ns}$. The relative stability of $\tau_{a v}$. lifetime in these probes is counterbalanced by decreased input from the second component. Thus, the $I_{3}$ intensity is unchanged or even slightly increased in dry-milled samples (see Table 1), resulting in equivalent growth (albeit not strongly evident) in fractional free volume $f_{v}$ (see Table 2).

Assuming two inputs contributing to an overall trapping in the studied glassy g-As-Se arising from distinct positron- and Ps-related traps, the reconstructed PAL spectra can be adequately described in terms of two-state STM expanded to accommodate second defect-related component instead of Ps-decay one (three-state additive STM) as was done in [39-41]. This approach should not be taken too seriously in view of typical resolution in reconstructed PAL spectra [39], but it is useful to analyze bulk positron lifetimes and compositional behavior of trapping rates in multichannel positron annihilation process [41]. The trapping modes for pelletized g-As $\mathrm{Se}_{100-\mathrm{x}}$ specimens (unmilled, dry-milled, and wet-milled ones) calculated in terms of three-state additive STM are given in Table 3.

Table 3. Trapping modes derived from unconstrained $x 3$-term decomposed PAL spectra of pelletized unmilled, dry-milled, and wet-milled $\mathrm{g}-\mathrm{As}_{\mathrm{x}} \mathrm{Se}_{100-\mathrm{x}}$ assuming three-state additive STM.

\begin{tabular}{|c|c|c|c|c|}
\hline Samples & $\tau_{B}^{1-2}$ & $\kappa_{d 1}$ & $\kappa_{d 2}$ & $\kappa_{d 1} / \kappa_{d 2}$ \\
\hline $\mathrm{g}-\mathrm{As}_{\mathrm{x}} \mathrm{Se}_{100-\mathrm{x}} \mathrm{x}$ & ns & $\mathrm{ns}^{-1}$ & $\mathrm{~ns}^{-1}$ & a.u. \\
\hline Unmilled, $x=5$ & 0.251 & 0.59 & 0.05 & 11.8 \\
\hline Dry-milled, $x=5$ & 0.249 & 0.70 & 0.05 & 14.0 \\
\hline Wet-milled, $x=5$ & 0.291 & 0.82 & 0.47 & 1.74 \\
\hline Unmilled, $x=10$ & 0.250 & 0.58 & 0.05 & 11.6 \\
\hline Dry-milled, $x=10$ & 0.251 & 0.68 & 0.05 & 13.6 \\
\hline Wet-milled, $x=10$ & 0.279 & 0.93 & 0.44 & 2.11 \\
\hline Unmilled, $x=20$ & 0.253 & 0.67 & 0.06 & 11.2 \\
\hline Dry-milled, $x=20$ & 0.252 & 0.64 & 0.06 & 10.7 \\
\hline Wet-milled, $x=20$ & 0.277 & 0.77 & 0.36 & 2.14 \\
\hline Unmilled, $x=29$ & 0.254 & 0.73 & 0.05 & 14.6 \\
\hline Dry-milled, $x=29$ & 0.256 & 0.70 & 0.05 & 14.0 \\
\hline Wet-milled, $x=29$ & 0.257 & 0.78 & 0.18 & 4.33 \\
\hline Unmilled, $x=40$ & 0.273 & 1.48 & 0.03 & 49.3 \\
\hline Dry-milled, $x=40$ & 0.273 & 1.22 & 0.07 & 17.4 \\
\hline Wet-milled, $x=40$ & 0.282 & 0.95 & 0.22 & 4.32 \\
\hline Unmilled, $x=45$ & 0.274 & 1.72 & 0.04 & 43.0 \\
\hline
\end{tabular}


Table 3. Cont.

\begin{tabular}{|c|c|c|c|c|}
\hline Samples & $\tau_{B}^{1-2}$ & $\kappa_{d 1}$ & $\kappa_{d 2}$ & $\kappa_{d 1} / \kappa_{d 2}$ \\
\hline $\mathrm{g}-\mathrm{As} \mathrm{s}_{\mathrm{x}} \mathrm{Se}_{100-\mathrm{x}}, \mathrm{x}$ & ns & $\mathrm{ns}^{-1}$ & $\mathrm{~ns}^{-1}$ & a.u. \\
\hline Dry-milled, $x=45$ & 0.273 & 1.18 & 0.07 & 16.9 \\
\hline Wet-milled, $x=45$ & 0.283 & 1.25 & 0.21 & 5.95 \\
\hline Unmilled, $x=50$ & 0.275 & 1.18 & 0.07 & 16.9 \\
\hline Dry-milled, $x=50$ & 0.277 & 1.10 & 0.06 & 18.3 \\
\hline Wet-milled, $x=50$ & 0.277 & 1.06 & 0.21 & 5.05 \\
\hline Unmilled, $x=55$ & 0.271 & 1.09 & 0.06 & 18.2 \\
\hline Dry-milled, $x=55$ & 0.272 & 1.38 & 0.07 & 19.7 \\
\hline Wet-milled, $x=55$ & 0.287 & 1.18 & 0.29 & 4.07 \\
\hline Unmilled, $x=60$ & 0.270 & 1.37 & 0.07 & 22.8 \\
\hline Dry-milled, $x=60$ & 0.274 & 1.19 & 0.07 & 17.0 \\
\hline Wet-milled, $x=60$ & 0.291 & 0.95 & 0.24 & 4.17 \\
\hline Unmilled, $x=65$ & 0.272 & 1.10 & 0.07 & 15.7 \\
\hline Dry-milled, $x=65$ & 0.259 & 1.32 & 0.07 & 18.9 \\
\hline Wet-milled, $x=65$ & 0.280 & 1.16 & 0.22 & 5.27 \\
\hline
\end{tabular}

Two types of principally different traps can be distinguished in the studied nanocomposites, these being short- and long-lived ones with $\kappa_{d 1}$ and $\kappa_{d 2}$ trapping rates, respectively $\left(\kappa_{d 1}>\kappa_{d 2}\right)$. In transition from unmilled to dry-milled probes, the latter are nearly unchangeable, possessing the $\kappa_{d 2}$ rate at the level of $\sim 0.05-0.07 \mathrm{~ns}^{-1}$. Under this transition, the former traps show a clearly decreasing tendency in their trapping rate $\kappa_{d 1}$ ascribed to the process of void agglomeration II schematically illustrated in Figure 1a (apart from compositions at the edges of the glass-forming region). The defect-free bulk positron lifetime $\tau_{B}{ }^{1-2}$ in these probes approaches $\sim 0.25-0.27 \mathrm{~ns}$, confirming that annihilating positrons are trapped in intrinsic FVE stabilized in g-As-Se environment [48-53].

Under transition to wet-milled arsenoselenides, the role of long-lived positron trapping sites is enormously enhanced so that their trapping rate $\kappa_{d 2}$ becomes only $2-5$ times less as $\kappa_{d 1}$ (see Table 3). The defect-free bulk positron lifetime $\tau_{B}{ }^{1-2}$ is also enriched in the pelletized samples of g-As $\mathrm{Se}_{100-\mathrm{x}} / \mathrm{PVP}$ nanocomposites to approximately $\sim 0.28-0.29 \mathrm{~ns}$. Hence, the medium where positron annihilation occurs is cardinally changed in wet-milled glassy arsenoselenides, being transferred rather to the PVP solution.

\subsection{Volumetric Nanostructurization in Dry-Milled and Wet-Milled $g-A s_{x} S e_{100-x}$ in Terms of Ps-to-Positron Trapping Conversion ( $x 3-x 2-C D A)$}

If processes of Ps-decaying and positron $\left\{\mathrm{e}^{+}\right\}$-trapping are interconnected so that no changes occur in other alternative channels, unconstrained three-term decomposed PAL spectra can be treated employing the formalism of recently developed x3-x2-CDA [21-23]. With this in mind, let us reconstruct the expected and most plausible positron annihilation channels in the studied $\mathrm{g}-\mathrm{As}_{\mathrm{x}} \mathrm{Se}_{100-\mathrm{x}}$-based nanocomposites using the respective matrix shown in Table 4.

Annihilation from defect-free bulk (DFB) states is assumed to be the most relevant and completely invariant process in all glassy arsenoselenides g-As-Se, whatever their technology. In Table 4, this channel of an intrinsic positron annihilation is denoted as $\{\mathrm{DFB}\}^{g-\mathrm{As}-\mathrm{Se}}$-int for all groups of samples (bulk, cgp, and fgp; unmilled and milled; pelletized and unpelletized). 
Table 4. Matrix of expected positron annihilation channels in $\mathrm{g}-\mathrm{As}_{\mathrm{x}} \mathrm{Se}_{100-\mathrm{x}}$-based composites.

\begin{tabular}{|c|c|c|c|c|}
\hline $\begin{array}{c}\text { Positron } \\
\text { Annihilation } \\
\text { Channel }\end{array}$ & $\begin{array}{l}\text { Bulk g-As-Se } \\
\text { (Unmilled, } \\
\text { Unpelletized) }\end{array}$ & $\begin{array}{l}\text { Cgp g-As-Se } \\
\text { (Unmilled, } \\
\text { Pelletized) }\end{array}$ & $\begin{array}{l}\text { Fgp g-As-Se } \\
\text { (Dry-Milled, } \\
\text { Pelletized) }\end{array}$ & $\begin{array}{c}\text { Fgp g-As-Se/PVP } \\
\text { (Wet-Milled, } \\
\text { Pelletized) }\end{array}$ \\
\hline$\{\mathrm{DFB}\}$ state & $\{\mathrm{DFB}\}^{\mathrm{g}-\mathrm{As}-\mathrm{Se}}$-int & $\{\mathrm{DFB}\}^{\mathrm{g}-\mathrm{As}-\mathrm{Se}}$-int & $\{\mathrm{DFB}\}^{\mathrm{g}-\mathrm{As}-\mathrm{Se}}$-int & $\{\mathrm{DFB}\}^{\mathrm{g}-\mathrm{As}-\mathrm{Se}}$-int \\
\hline \multirow{2}{*}{$\begin{array}{c}\text { Positron } \\
\left\{\mathrm{e}^{+}\right\} \text {-traps }\end{array}$} & $\left\{\mathrm{e}^{+}\right\}^{\mathrm{g}-\mathrm{As}-\mathrm{Se}}-$ int & $\left\{\mathrm{e}^{+}\right\}^{\mathrm{g}-\mathrm{As}-\mathrm{Se}}-$ int & $\left\{\mathrm{e}^{+}\right\}^{\mathrm{g}-\mathrm{As}-\mathrm{Se}}-$ int & $\left\{\mathrm{e}^{+}\right\}^{\mathrm{g}-\mathrm{As}-\mathrm{Se}}-\mathrm{int}$ \\
\hline & - & $\left\{\mathrm{e}^{+}\right\}^{\mathrm{g}-\mathrm{As}-\mathrm{Se}}-\mathrm{IF}-1$ & $\left\{\mathrm{e}^{+}\right\}^{\mathrm{g}-\mathrm{As}-\mathrm{Se}}-\mathrm{IF}-2$ & $\left\{\mathrm{e}^{+}\right\}^{\mathrm{g}-\mathrm{As}-\mathrm{Se} / \mathrm{PVP}}-\mathrm{IF}-3$ \\
\hline \multirow{2}{*}{$\begin{array}{l}\text { Positronium } \\
\{\text { Ps\}-traps }\end{array}$} & $\{\mathrm{Ps}\}^{\mathrm{g}-\mathrm{As}-\mathrm{Se}}$-int-1 & $\{\text { Ps }\}^{\text {g-As-Se}}$-int-2 & $\{\mathrm{Ps}\}^{\mathrm{g}-\mathrm{As}-\mathrm{Se}}-$ int-2 & $\{\mathrm{Ps}\}^{\mathrm{g}-\mathrm{As}-\mathrm{Se}}-\mathrm{int}-2$ \\
\hline & - & $\{\text { Ps }\}^{\text {g-As-Se_FVE-1 }}$ & $\{\text { Ps }\}^{g-A s-S e}-F V E-2$ & $\{\mathrm{Ps}\}^{\mathrm{g}-\mathrm{As}-\mathrm{Se} / \mathrm{PVP}}$ _FVE-3 \\
\hline
\end{tabular}

Notes: DFB-defect-free bulky (state); int-intrinsic; GB-grain-boundary; IF-interfacial; cgp-coarse-grained powdered; fgp-fine-grained powdered; equivalent annihilation channels are yellow-colored; positron-Ps trapping-conversion channels are grey-colored.

Annihilation from positron ( $\mathrm{e}^{+}$)-traps (positron trapping) is an alternative path of free positrons used to annihilate with electrons through defective states acting as sites capturing positrons. One group of these $\left(\mathrm{e}^{+}\right)$-traps, denoted in Table 4 as $\left\{\mathrm{e}^{+}\right\}$g-As-Se - int, is connected with intrinsic sub-nm-scaled FVE proper to glassy arsenoselenides. The most effective positron trapping centers of this type are multiatomic vacancies stabilized in the g-As-Se network at the bottom of $\mathrm{AsSe}_{3 / 2}$ pyramids in a preferentially negative environment of chalcogen (Se) atoms [48-53]. Another group of positron traps (denoted in Table 4 as $\left.\left\{\mathrm{e}^{+}\right\}^{\mathrm{g}-\mathrm{As}-\mathrm{Se}}-\mathrm{IF}-1,2,3\right)$ is character to fgp g-As-Se, these FVE being stabilized under pelletization [61] as GB and TJ (i.e., intersection between three or more adjoining NP) in samples of different milling, pre-history [62-66].

Annihilation from Ps-decaying states ascribed to nanostructured FVE (free-volume sub-nm-scaled holes, voids, pores, cavities, etc.) dominates in sub-nanometric porous structures by interaction with electrons from the environment, owing to the "pick-off" process $[18,24,32]$. One part of these defects (denoted in Table 4 as $\left.\{\mathrm{Ps}\}\}^{g-A s-S e}-i n t-1,2\right)$ are connected with decaying of bound positron-electron states (Ps atoms) in intrinsic imperfections of a glassy state stabilized under rapid melt-quenching. It can be reasonably assumed that this source of Ps-decaying site (yellow-colored in Table 4) is completely analogous in all pelletized samples. Another part of the Ps-decaying site is related to FVE, marked in Table 4 as $\{\mathrm{Ps}\}\}^{g-A s-S e}$-FVE-1,2,3, which appears specifically in the powdered glassy arsenoselenides owing to material attrition on the finest nanometer pieces. These FVE represent IF free-volume spaces between contacting NP (such as disclinations or TJ between adjoining NP), where both conditions stabilizing Ps-related states $[24,32]$ are satisfied. For metallic multi-particulate systems, the most essential excess of additional free volume is expected with individual crystallites less than 5-10 nm [62-65].

Under this consideration, it is evident that conversion should be expected in the respective channels in dry-milled and wet-milled samples (grey-colored in Table 4), involving changes connected with positron trapping from $\left\{\mathrm{e}^{+}\right\}^{\mathrm{g}-\mathrm{As}-\mathrm{Se}}-\mathrm{IF} / \mathrm{GB}-2$ to $\left\{\mathrm{e}^{+}\right\}^{\mathrm{g}-\mathrm{As}-\mathrm{Se}} / \mathrm{PVP}$

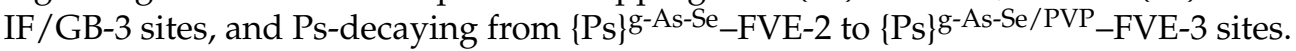

The calculated trapping modes for dry-milled glassy arsenoselenides g-As $\mathrm{Se}_{100-\mathrm{x}}$ defined in respect to iso-compositional g- $\mathrm{As}_{\mathrm{x}} \mathrm{Se}_{100-\mathrm{x}} / \mathrm{PVP}$ nanocomposites, employing formalism of x3-x2-CDA [21-23], are gathered in Table 5 .

Positive intensities of both $I_{n}$ and $I_{\text {int }}$ components in the generalized x2-component PAL spectra reconstructed from respective unconstrained $x 3$-component spectra of pelletized dry-milled and wet-milled samples, along with well-defined component inputs $\left(\tau_{n} \cdot I_{n}\right)$ and $\left(\tau_{\text {int }} \cdot I_{\text {int }}\right)$, testify that $\{$ Ps\}-decay sites in wet-milled samples can be imagined as directly transformed to $\left\{\mathrm{e}^{+}\right\}$-traps in dry-milled ones. The derived values of defect-related positron lifetimes at the level of $\tau_{\text {int }}=0.36-0.38 \mathrm{~ns}$ testify that $\left\{\mathrm{e}^{+}\right\}$-traps are extended IF defects, close in their volumes to di- and/or tri-atomic vacancies having $\sim 0.25-0.28 \mathrm{~nm}$ in radius (stabilized at the bottom of $\mathrm{AsSe}_{3 / 2}$ pyramids in negative environment of $\mathrm{Se}$ atoms), as it follows from the known semi-empirical relationship for these glasses [51-53]. This finding agrees well with $\left(\tau_{\text {int }}-\tau_{b}{ }^{N P}\right)=0.09-0.11 \mathrm{~ns}$ and $\tau_{\text {int }} / \tau_{b}{ }^{N P}=1.34-1.46$ as indicatives of these vacancy-type defects [18]. Both parameters are slightly increased as going towards under-stoichiometric Se-rich glassy arsenoselenides $\mathrm{g}-\mathrm{As}_{\mathrm{x}} \mathrm{Se}_{100-\mathrm{x}}$ along with 
decreased trapping rate in defects $\kappa_{d}{ }^{N P}$ (see Table 5), testifying that $\mathrm{AsSe}_{3 / 2}$ pyramids are destroyed by an abundant amount of homonuclear Se-Se covalent chemical bonds (causing void agglomeration II, as schematically depicted in Figure 1a).

Table 5. Calculated $x 3-x 2-C D A$ trapping modes in dry-milled $g-\mathrm{As}_{\mathrm{x}} \mathrm{Se}_{100-\mathrm{x}}$ defined in respect to iso-compositional wet-milled $\mathrm{g}-\mathrm{As}_{\mathrm{x}} \mathrm{Se}_{100-\mathrm{x}} / \mathrm{PVP}$ nanocomposites.

\begin{tabular}{|c|c|c|c|c|c|c|c|c|c|}
\hline \multirow[t]{2}{*}{ Sample } & \multicolumn{2}{|c|}{ I Component } & \multicolumn{2}{|c|}{ II Component } & \multicolumn{5}{|c|}{ Positron Trapping Modes } \\
\hline & $\tau_{n}$ & $I_{n}$ & $\tau_{\text {int }}$ & $I_{\text {int }}$ & $\tau_{a v} N P$ & $\tau_{b}{ }^{N P}$ & $\kappa_{d}^{N P}$ & $\tau_{\text {int }}-\tau_{b}{ }^{N P}$ & $\tau_{\text {int }} / \tau_{b}{ }^{N P}$ \\
\hline & ns & a.u. & ns & a.u. & ns & ns & $\mathrm{ns}^{-1}$ & ns & a.u. \\
\hline$x=5$ & 0.210 & 0.573 & 0.357 & 0.310 & 0.261 & 0.245 & $0.69 \cong 0.7$ & 0.11 & 1.46 \\
\hline$x=10$ & 0.218 & 0.574 & 0.364 & 0.272 & 0.265 & 0.250 & $0.60 \cong 0.6$ & 0.11 & 1.46 \\
\hline$x=20$ & 0.216 & 0.561 & 0.357 & 0.201 & 0.264 & 0.249 & $0.63 \cong 0.6$ & 0.11 & 1.43 \\
\hline$x=33$ & 0.212 & 0.475 & 0.362 & 0.304 & 0.270 & 0.253 & $0.77 \cong 0.8$ & 0.11 & 1.44 \\
\hline$x=40$ & 0.194 & 0.334 & 0.352 & 0.472 & 0.287 & 0.263 & $1.35 \cong 1.4$ & 0.09 & 1.34 \\
\hline$x=45$ & 0.205 & 0.320 & 0.369 & 0.376 & 0.294 & 0.270 & $1.17 \cong 1.2$ & 0.10 & 1.37 \\
\hline$x=50$ & 0.211 & 0.351 & 0.368 & 0.403 & 0.295 & 0.274 & $1.08 \cong 1.1$ & 0.10 & 1.35 \\
\hline$x=55$ & 0.193 & 0.331 & 0.358 & 0.464 & 0.289 & 0.264 & $1.39 \cong 1.4$ & 0.09 & 1.35 \\
\hline$x=60$ & 0.201 & 0.359 & 0.367 & 0.418 & 0.291 & 0.266 & $1.21 \cong 1.2$ & 0.10 & 1.38 \\
\hline$x=65$ & 0.193 & 0.315 & 0.353 & 0.433 & 0.286 & 0.261 & $1.37 \cong 1.4$ & 0.09 & 1.35 \\
\hline
\end{tabular}

In a particular case of dry-milled $\mathrm{g}-\mathrm{As}_{2} \mathrm{Se}_{3}$, positron $\left\{\mathrm{e}^{+}\right\}$-trapping channel substitutes $\{$ Ps $\}$-decaying contribution from empty inter-particulate junctions with $\tau_{3}=2.087 \mathrm{~ns}$ by input from PVP-filled ones with $\tau_{3}=1.891$ ns (Table 5). The governing trapping-conversion process, i.e., appearance of positron $\left\{\mathrm{e}^{+}\right\}$-traps with defect-related lifetime $\tau_{\text {int }}=0.352 \mathrm{~ns}$ instead of Ps-decaying sites with $\tau_{3}=2.087 \mathrm{~ns}$, occurs in the environment possessing defect-free bulk lifetime $\tau_{b}{ }^{N P}=0.263 \mathrm{~ns}$ (Table 5). This value is evidently above the bulk lifetime in crystalline $\mathrm{c}-\mathrm{As}_{2} \mathrm{Se}_{3}\left(\tau_{b}=0.240 \mathrm{~ns}\right.$ [47]), but below $\tau_{b}$ in $\mathrm{g}-\mathrm{As}_{2} \mathrm{Se}_{3}(\sim 0.280-0.285 \mathrm{~ns}$ as determined from unconstrained x2-term decomposed PAL spectra ignoring input from Ps-decaying [50,51]). Thus, the chemical environment where Ps-to-positron trapping conversion occurs, i.e., inner spaces where $\left\{\mathrm{e}^{+}\right\}$-traps appear (in dry-milled $\mathrm{g}-\mathrm{As}_{2} \mathrm{Se}_{3}$ ) instead of Ps-decaying sites (in PVP-capped $\left.g-\mathrm{As}_{2} \mathrm{Se}_{3}\right)$, is indeed glassy arsenoselenide matrix.

Concerning the $x 3-x 2-C D A$, we are grounded on the assumption that the process of Ps-to-positron trapping conversion is indeed the alone process linking dry-milled and wet-milled glassy arsenoselenides. This condition is satisfied, owing to nearly the same channel of positron-electron annihilation originating from DFB states in all $\mathrm{g}-\mathrm{As}_{\mathrm{x}} \mathrm{Se}_{100-\mathrm{x}}$ samples (mainly, due to a small amount of PVP in wet-milled nanocomposites).

\subsection{Positronics of Nanostructurization-Driven Volumetric Effects in $g-A s_{x} S e_{100-x}$}

Thus, the most striking feature of comparative PAL-spectra analysis in NM-modified glassy As-Se is an evidently decreasing trend in contribution to o-Ps decaying modes in the row of pelletized samples from PVP to wet-milled $\mathrm{As}_{\mathrm{x}} \mathrm{Se}_{100-\mathrm{x}}$, and further to isocompositional dry-milled and unmilled specimens. Such variations with nanostructurallyand compositionally-tuned third-component contribution and average positron lifetime $\tau_{a v}$ compose an interesting specificity in Positronics, revealed in monotonic behavior of both PAL spectrum peaks and PAL spectrum "tails", which can be highlighted as "rainbow" effects. A group of nanostructurization-tuned "rainbow" effects is spectacularly well revealed in reconstructed PAL spectra of studied $\mathrm{As}_{\mathrm{x}} \mathrm{Se}_{100-\mathrm{x}}$ (Figures 5 and 6). 

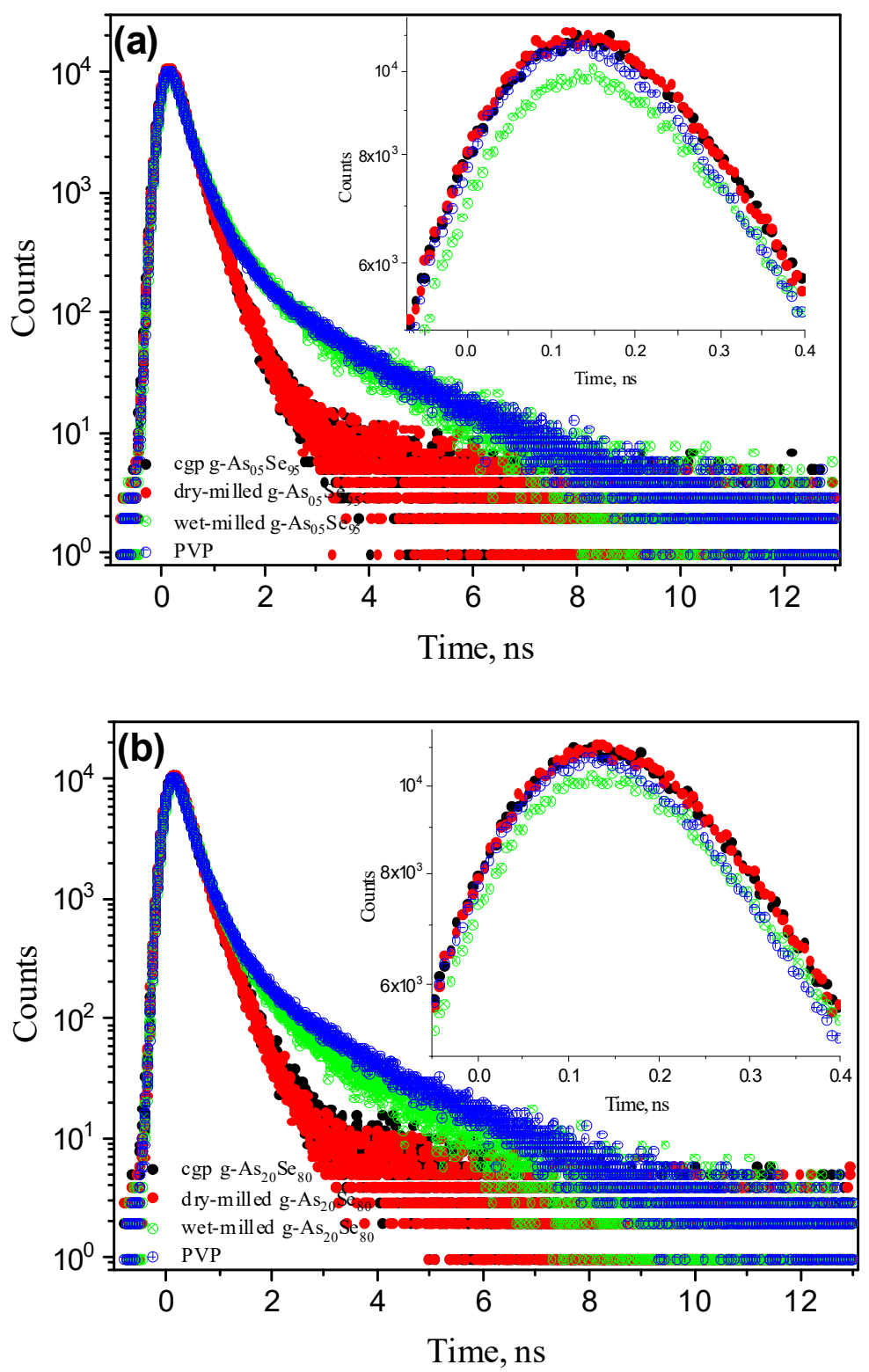

Figure 5. Nanostructurization-tuned "rainbow" effects in under-stoichiometric g-As ${ }_{5} \mathrm{Se}_{95}$ (a) and g- $\mathrm{As}_{20} \mathrm{Se}_{80}$ (b) pellets: the PAL spectra were collected for unmilled (black points), dry-milled (red points), and wet-milled glassy arsenoselenides (green points) and compared with PAL spectrum of PVP (blue points). The insert shows respective PAL spectra peaks of wet-milled probes, abruptly depressed in both wings and slightly shifted to higher lifetimes due to enhanced Ps-formation probability and increased mean positron lifetime $\tau_{a v}$. The empty gap between PAL spectra "tails" for dry-milled and wet-milled samples is due to jump in hole density of o-Ps decaying states in transition to PVP medium.

In over-stoichiometric g-As ${ }_{50} \mathrm{Se}_{50}$ and $\mathrm{g}$ - $\mathrm{As}_{60} \mathrm{Se}_{40}$ probes affected to $\mathrm{NM}$, nanostructurizationtuned "rainbow" effects in the PAL spectra are revealed less specifically (Figure 6a,b). Owing to moderated Ps-formation probability and decreased average lifetime, these nanocomposites demonstrate a nearly invariant trend in the PAL spectrum peak, which is mainly depressed in the right wing after wet NM. Nevertheless, the PAL spectra "tails" are expanded in transition from unmilled and dry-milled specimens to PVP-capped ones without distinct empty gaps on the histogram of accumulated annihilation events (the changes in $f_{v}$ for dry-milled and wet-milled $\mathrm{g}-\mathrm{As}_{60} \mathrm{Se}_{40}$ reach only $\sim 0.67 \%$, see Table 2 ). 

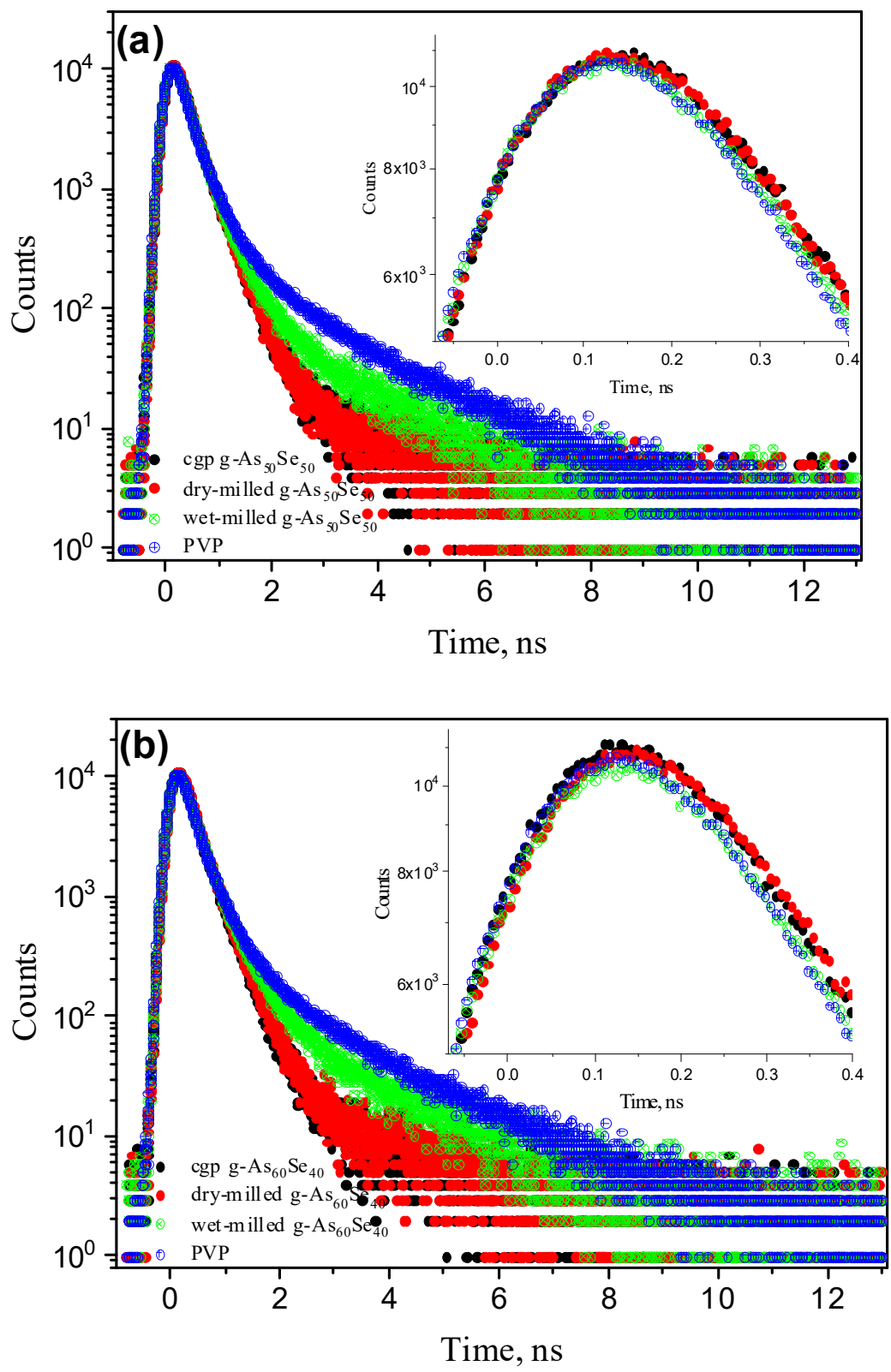

Figure 6. Nanostructurization-tuned "rainbow" effects in over-stoichiometric g-As ${ }_{50} \mathrm{Se}_{50}(\mathbf{a})$ and g-As ${ }_{60} \mathrm{Se}_{40}$ (b) pellets: the PAL spectra were collected for unmilled (black points), dry-milled (red points), and wet-milled glassy arsenoselenides (green points) and compared with the PAL spectrum of PVP (blue points). The insert shows nearly invariant tendency in the PAL spectra peaks depressed in the right wing after wet nanomilling due to moderated Ps-formation probability and slightly changed average positron lifetime $\tau_{a v}$. Changes in the PAL spectra "tails" for unmilled, dry-milled, and wet-milled probes is owing to increase in the hole density of o-Ps decaying states. There is no empty gap between PAL spectra "tails" for dry-milled and wet-milled samples caused by changes in o-Ps decaying states in transition to the PVP medium.

The group of compositionally tuned "rainbow" effects are detectable in the PAL spectra of $\mathrm{g}-\mathrm{As}_{\mathrm{x}} \mathrm{Se}_{100-\mathrm{x}}$ affected to NM in a wet mode (viz. g-As $\mathrm{Se}_{100-\mathrm{x}} / \mathrm{PVP}$ nanocomposites) with a monotonically changed $x$ parameter (see Figure 7 ).

These effects are more pronounced in under-stoichiometric arsenoselenides $(x<40$, Figure 7a), demonstrating (with going to "pure" Se) a clearly depressing tendency in the PAL spectrum peak (symmetric in both left and right wings) because of an increased average positron lifetime $\left(\tau_{a v}.\right)$, without changes in the peak position, in view of negligible 
variations in the long-lived $\tau_{3}$ lifetime (see Table 1 ). The widely expanded "tails" in the PAL spectra of under-stoichiometric nanocomposites in Figure 7a correspond to rich diversity in contribution of a long-lived Ps-related component defined by $I_{3}$ intensity (the observed changes from 0.052 for $\mathrm{g}-\mathrm{As}_{40} \mathrm{Se}_{60} / \mathrm{PVP}$ to 0.112 for $\mathrm{g}-\mathrm{As}_{5} \mathrm{Se}_{95} / \mathrm{PVP}$, see Table 1$)$. In contrast, in over-stoichiometric arsenoselenides $(x>40)$, where intensities of a long-lived Ps-related component $\left(I_{3}\right)$ are only slightly scattered around $I_{3} \sim 0.05$, the compositionally tuned "rainbow" effects are more suppressed (see Figure 7b).
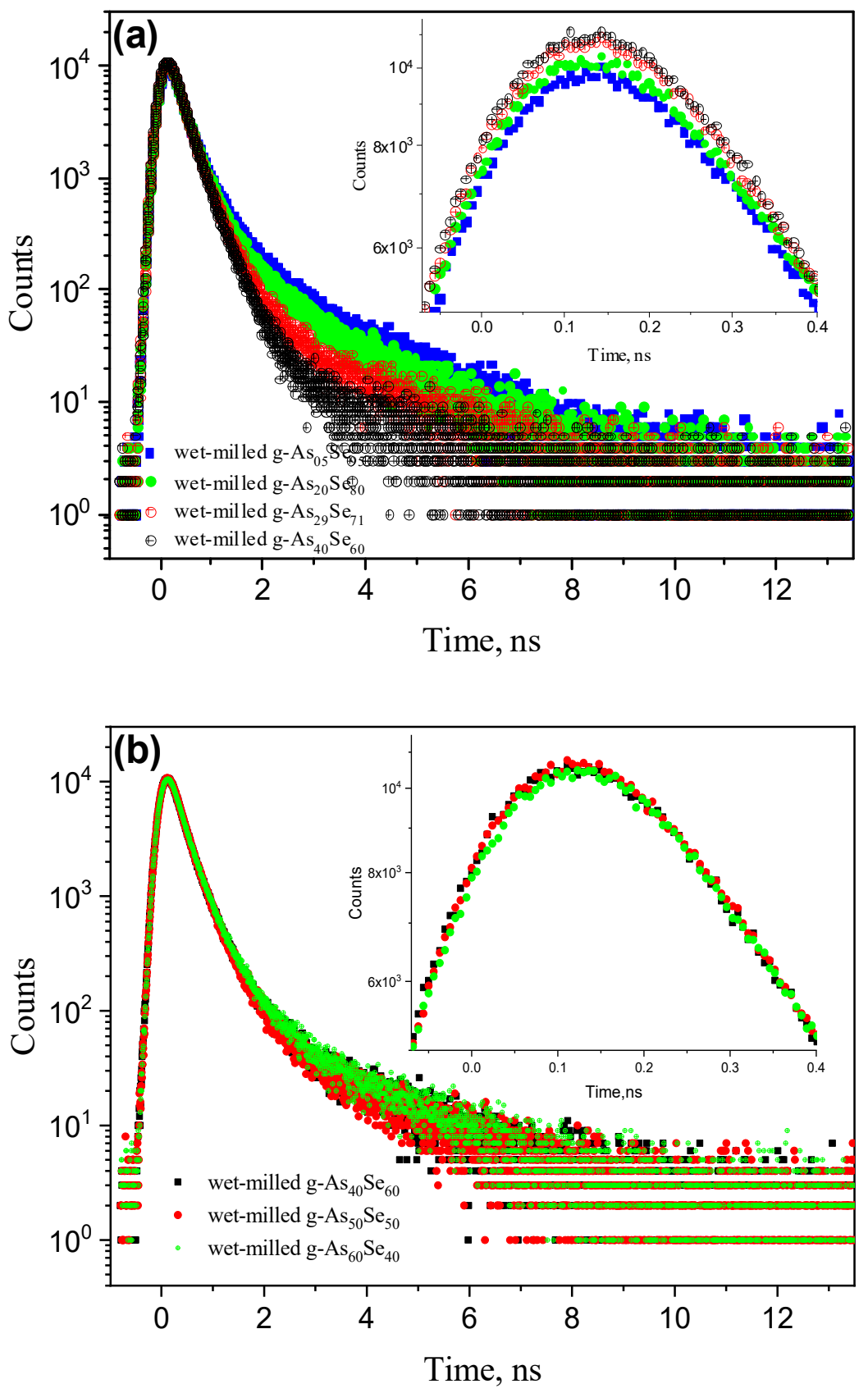

Figure 7. Compositionally tuned "rainbow" effects in PVP-capped g-As $\mathrm{Se}_{\mathrm{x}} \mathrm{Se}_{-\mathrm{x}}$ pellets: (a)—understoichiometric samples of $\mathrm{g}-\mathrm{As}_{5} \mathrm{Se}_{95}$ (blue points), g- $\mathrm{As}_{20} \mathrm{Se}_{80}$ (green points), g- $\mathrm{As}_{29} \mathrm{Se}_{71}$ (red points) and stoichiometric g-As ${ }_{40} \mathrm{Se}_{60}$ (black points); (b)—stoichiometric samples of g-As ${ }_{40} \mathrm{Se}_{60}$ (black points) and over-stoichiometric g-As ${ }_{50} \mathrm{Se}_{50}$ (red points) and $\mathrm{g}-\mathrm{As}_{60} \mathrm{Se}_{40}$ (green point). The inserts show peak-changing trends in the PAL spectra of PVP-capped samples due to changes in average positron lifetime $\tau_{a v}$ and long-lived component intensity $I_{3}$. 


\section{Conclusions}

Volumetric effects, involving changes in atomic-deficient free-volume structure, were studied in glassy arsenoselenides g-As $\mathrm{Se}_{100-\mathrm{x}}$ subjected to high-energy dry and wet milling (in $0.5 \%$ PVP water solution) employing the method of positron annihilation lifetime (PAL) spectroscopy.

Preliminary analysis of milling-driven volumetric nanostructurization in $\mathrm{g}-\mathrm{As}_{\mathrm{x}} \mathrm{Se}_{100-\mathrm{x}}$ was performed with a modified two-state simple trapping model applied under slight input from a Ps-decay channel. From this analysis, it is proved that additional positron trapping sites appear in bulky over-stoichiometric $g-\mathrm{As}_{\mathrm{x}} \mathrm{Se}_{100-\mathrm{x}}(\mathrm{x}>40)$ under coarse powdering, followed by a conventional pelletization procedure. In contrast, slight agglomeration of large positron traps is expected in transition from unmilled to dry-milled glassy arsenoselenides. In comparison with coarse-grained powdered and dry-milled arsenoselenides, positron trapping in wet-milled probes, representing themselves as $\mathrm{g}-\mathrm{As}_{\mathrm{x}} \mathrm{Se}_{100-\mathrm{x}} / \mathrm{PVP}$ nanocomposites, was essentially modified by enriched contributions from Ps-decaying states stabilized in a "pure" PVP environment.

These findings were proved, assuming two inputs contributing to trapping in $\mathrm{g}$ $\mathrm{As}_{\mathrm{x}} \mathrm{Se}_{100-\mathrm{x}}$ arising from distinct positron- and Ps-related sites (three-state additive simple trapping model). It is confirmed that annihilating positrons in dry-milled probes are trapped in free-volume defects stabilized intrinsically in a g-As-Se environment; however, the medium where positron annihilation occurs is cardinally changed in wet-milled probes being replaced by PVP solution.

Formalism of x3-x2-CDA (coupling decomposition algorithm) describing the conversion of bound positron-electron (positronium, Ps) states into positron traps was applied to identify free volume nanostructurization in pelletized PVP-capped g-As-Se nanocomposites, with respect to iso-compositional dry-milled ones. Under wet milling, Ps-decaying sites stabilized in inter-nanoparticulate junctions with the PVP environment replaced positron traps in dry-milled samples having defect-specific lifetimes of $\sim 0.36-0.38 \mathrm{~ns}$, the latter corresponding to multi-atomic vacancies near trigonal $\mathrm{AsSe}_{3 / 2}$ pyramids in a mostly negative environment of bridging Se atoms. Tending to under-stoichiometric Se-rich glassy arsenoselenides, $\mathrm{g}-\mathrm{As}_{\mathrm{x}} \mathrm{Se}_{100-\mathrm{x}}$, these positron-trapping sites are agglomerated due to an abundant amount of homonuclear Se-Se bonds.

As a final accord in positronics, the genesis of reconstructed three-component PAL spectra with nanostructurally- and compositionally-tuned Ps-decay contribution and average positron lifetime is considered as the foundation of specific "rainbow" effects observed in a row of these spectra for pelletized PVP, and further, wet-milled, dry-milled, and unmilled $\mathrm{g}-\mathrm{As}_{\mathrm{x}} \mathrm{Se}_{100-\mathrm{x}}$.

Author Contributions: Conceptualization, O.S., B.B., P.B., Y.S.; methodology, Y.S., C.B.-P. and Z.L.B.; validation, O.S., B.B. and P.B.; formal analysis, A.I., B.M. and Z.L.B.; investigation, Y.S., B.M., C.B.-P. and A.I.; writing—original draft preparation, O.S.; writing—review and editing, Y.S., P.B.; visualization, Y.S. and B.M. All authors have read and agreed to the published version of the manuscript.

Funding: The work is supported by the Slovak Research and Development Agency under contract no. APVV-18-0357 and the Slovak Grant Agency VEGA under contract no. 2/0044/18 (Z.L.B. and P.B.). The project is co-financed by the Polish National Agency for Academic Exchange (project code PPN/BFR/2020/1/00043) and French Ministries of Europe and Foreign Affairs and Higher Education, Research and Innovation (project code 46893NA) in the frame of the Programme Hubert Curien PHC POLONIUM (Y.S., B.M., C.B., B.B.).

Institutional Review Board Statement: Not applicable.

Informed Consent Statement: Not applicable.

Data Availability Statement: The data presented in this study are available upon request from the corresponding author.

Conflicts of Interest: The authors declare no conflict of interest. 


\section{References}

1. Adam, J.-L.; Zhang, X. Chalcogenide Glasses: Preparation, Properties and Application; Woodhead Publishing Series in Electronic and Optical Mater: Philadelphia, PA, USA, 2013; pp. 1-717.

2. Feltz, A. Amorphous Inorganic Materials and Glasses; VCH: Weinheim, Germany, 1993; pp. 1-446.

3. Cui, S.; Chahal, R.; Shpotyuk, Y.; Boussard, C.; Lucas, J.; Charpentier, F.; Tariel, H.; Loreal, O.; Nazabal, V.; Sire, O.; et al. Selenide and telluride glasses for mid-infrared bio-sensing. Proc. SPIE 2014, 8938, 893805. [CrossRef]

4. Dilda, P.J.; Hogg, P.J. Arsenical-based cancer drugs. Cancer Treat. Rev. 2007, 33, 542-564. [CrossRef] [PubMed]

5. Ding, W.; Zhang, L.; Kim, S.; Tian, W.; Tong, Y.; Liu, J.; Ma, Y.; Chen, S. Arsenic sulfide as a potential anti-cancer drug. Molec. Med. Rep. 2015, 11, 968-974. [CrossRef]

6. Tian, Y.; Wang, X.; Xi, R.; Pan, W.; Jiang, S.; Li, Z.; Zhao, Y.; Gao, G.; Liu, D. Enhanced antitumor activity of realgar mediated by milling it to nanosize. Intern. J. Nanomed. 2014, 9, 745-757. [CrossRef]

7. $\mathrm{Wu}$, J.-Z.; Ho, P.C. Evaluation of the in vitro activity and in vivo bioavailability of realgar nanoparticles prepared by cryo-grinding. Eur. J. Pharm. Sci. 2006, 29, 35-44. [CrossRef]

8. Bujnakova, Z.; Balaz, P.; Makreski, P.; Jovanovski, G.; Caplovicova, M.; Caplovic, L.; Shpotyuk, O.; Ingram, A.; Lee, T.-C.; Cheng, J.-J.; et al. Arsenic sulfide nanoparticles prepared by milling: Properties, free-volume characterization, and anti-cancer effects. J. Mater. Sci. 2015, 50, 1973-1985. [CrossRef]

9. Shpotyuk, O.; Bujňáková, Z.; Baláž, P.; Ingram, A.; Demchenko, P.; Kovalskiy, A.; Vlcek, M.; Shpotyuk, Y.; Cebulski, J.; Dziedzic, A. Nanostructurization effects in PVP-stabilized tetra-arsenic tetrasulfide $\mathrm{As}_{4} \mathrm{~S}_{4}$ nanocomposites. Mater. Chem. Phys. 2017, 186, 251-260. [CrossRef]

10. Baláž, P.; Achimovicova, M.; Baláž, M.; Billik, P.; Cherkezova-Zheleva, Z.; Manuel Criado, J.; Delogu, F.; Dutkova, E.; Gaffet, E.; Gotor, F.J.; et al. Hallmarks of mechanochemistry: From nanoparticles to technology. Chem. Soc. Rev. 2013, 42, 7571-7637. [CrossRef]

11. Baláž, P.; Baláž, M.; Shpotyuk, O.; Demchenko, P.; Vlcek, M.; Shopska, M.; Briančin, J.; Bujňáková, Z.; Shpotyuk, Y.; Selepová, B.; et al. Properties of arsenic sulphide $\left(\beta-\mathrm{As}_{4} \mathrm{~S}_{4}\right)$ modified by mechanical activation. J. Mater. Sci. 2017, 52, 1747-1758. [CrossRef]

12. Boldyreva, E. Mechanochemistry of inorganic and organic systems: What is similar, what is different? Chem. Soc. Rev. 2013, 42, 7719-7738. [CrossRef] [PubMed]

13. Ghosh, G.; Naskar, M.K.; Patra, A.; Chatterjee, M. Synthesis and characterization of PVP-encapsulated ZnS nanoparticles. Opt. Mater. 2006, 28, 1047-1053. [CrossRef]

14. Saravanan, I.; Diwakar, S.; Mohankumar, R.; Pandurangan, A.; Jayavel, R. Synthesis, Structural and Optical Properties of PVP Encapsulated CdS Nanoparticles. Nanomater. Nanotechnol. 2011, 1, 42-48. [CrossRef]

15. Shpotyuk, O.; Ingram, A.; Shpotyuk, Y.; Bujňáková, Z.; Baláž, P. PVP-stabilized arsenic sulfide As $\mathrm{S}_{4}$ nanocomposites probed with positron annihilation lifetime spectroscopy. Polym. Eng. Sci. 2017, 57, 502-505. [CrossRef]

16. West, R.N. Positron studies of condensed matter. Adv. Phys. 1973, 22, 263-383. [CrossRef]

17. Saarinen, K.; Hautojarvi, P.; Corbel, C. Positron annihilation spectroscopy of defects in semiconductors. Semicond. Semimet. 1998, 51A, 210-285. [CrossRef]

18. Krause-Rehberg, R.; Leipner, H. Positron Annihilation in Semiconductors: Defect Studies; Springer: Berlin/Heidelberg, Germany, 1999; pp. 1-350.

19. Tuomisto, F.; Makkonen, I. Defect identification in semiconductors with positron annihilation: Experiment and theory. Rev. Mod. Phys. 2013, 85, 1583-1631. [CrossRef]

20. Shpotyuk, M.; Ingram, A.; Shpotyuk, O. Characterization of radiation-induced effects in amorphous arsenic sulfides by positron annihilation lifetime spectroscopy. J. Mater. Res. 2015, 30, 1422-1429. [CrossRef]

21. Shpotyuk, O.; Filipecki, J.; Ingram, A.; Golovchak, R.; Vakiv, M.; Klym, H.; Balitska, V.; Shpotyuk, M.; Kozdras, A. Positronics of subnanometer atomistic imperfections in solids as a high-informative structure characterization tool. Nanoscale Res. Lett. 2015, 10, 77. [CrossRef] [PubMed]

22. Shpotyuk, O.; Ingram, A.; Shpotyuk, Y. Free-volume characterization of nanostructurized substances by positron annihilation lifetime spectroscopy. Nucl. Instr. Meth. Phys. Res. 2018, 416, 102-109. [CrossRef]

23. Shpotyuk, Y.; Cebulski, J.; Ingram, A.; Shpotyuk, O. Mathematical modelling of elementary trapping-reduction processes in positron annihilation lifetime spectroscopy: Methodology of Ps-to-positron trapping conversion. J. Phys. 2017, 936, 012049. [CrossRef]

24. Jean, Y.C.; Mallon, P.E.; Schrader, D.M. Principles and Application of Positron and Positronium Chemistry; World Scientific: Singapore, 2003; pp. 1-406.

25. Kansy, J. Microcomputer program for analysis of positron annihilation lifetime spectra. J. Nucl. Instr. Meth. Phys. Res. 1996, 374, 235-244. [CrossRef]

26. Deng, Q.; Sundar, C.S.; Jean, Y.C. Pressure dependence of free-volume hole properties in an epoxy polymer. J. Phys. Chem. 1992, 96, 492-495. [CrossRef]

27. Dlubek, G.; Saarinen, K.; Fretwell, H.M. Positron states in polyethylene and polytetrafluoroethylene: A positron lifetime and Doppler-broadening study. Nucl. Instr. Meth. Phys. Res. 1998, 142, 139-155. [CrossRef] 
28. Dlubek, G.; Clarke, A.P.; Fretwell, H.M.; Dugdale, S.B.; Alam, M.A. Positron lifetime studies of free volume4 hole size distribution in glassy polycarbonate and polysterene. Phys. Stat. Sol. 1996, 157, 351-364. [CrossRef]

29. Pascual-Izarra, C.; Dong, A.W.; Pas, S.J.; Hill, A.J.; Boyd, B.J.; Drummond, C.J. Advanced fitting algorithms for analysing positron annihilation lifetime spectra. Nucl. Instr. Meth. Phys. Res. 2009, 603, 456-466. [CrossRef]

30. Shpotyuk, O.; Filipecki, J. Radiation-induced defects in vitreous chalcogenide semiconductors studied by positron annihilation method. Mater. Sci. Eng. 2002, 91-92, 537-540. [CrossRef]

31. Ghosh, S.; Nambissan, P.M.G.; Bhattacharya, R. Positron annihilation and Mössbauer spectroscopic studies of $\operatorname{In}^{3+}$ substitution effects in bulk and nanocrystalline $\mathrm{MgMn}_{0 \cdot 1} \mathrm{Fe}_{1 \cdot 9-x} \mathrm{In}_{x} \mathrm{O}_{4}$. Phys. Lett. 2004, 325, 301-308. [CrossRef]

32. Castelli, F.; Consolatti, G.; Marlotti, G.T. Positronium confined in nanocavities: The role of electron exchange correlations. Nanomaterials 2021, 11, 2350. [CrossRef] [PubMed]

33. Chakraverty, S.; Mitra, S.; Mandal, K.; Nambissan, P.M.G.; Chattopadhyay, S. Positron annihilation studies of some anomalous features of $\mathrm{NiFe}_{2} \mathrm{O}_{4}$ nanocrystals grown in $\mathrm{SiO}_{2}$. Phys. Rev. 2005, 71, 024115. [CrossRef]

34. Mitra, S.; Mandal, K.; Sinha, S.; Nambissan, P.M.G.; Kumar, S. Size and temperature dependent cationic redistribution in NiFe ${ }_{2} \mathrm{O}_{4}$ $\left(\mathrm{SiO}_{2}\right)$ nanocomposites: Positron annihilation and Mössbauer studies. J. Phys. 2006, 39, 4228-4235. [CrossRef]

35. Shpotyuk, O.; Ingram, A.; Filipecki, J.; Bujňáková, Z.; Baláž, P. Positron annihilation lifetime study of atomic imperfections in nanostructurized solids: On the parameterized trapping in wet-milled arsenic sulphides $\mathrm{As}_{4} \mathrm{~S}_{4}$. Phys. Stat. Sol. 2016, 253, 1054-1059. [CrossRef]

36. Shpotyuk, O.; Ingram, A.; Shpotyuk, O. Photopolymerization shrinkage in dimethacrylate-based dental restorative composites probed by means of positron annihilation lifetime spectroscopy. Polymer 2020, 196, 122485. [CrossRef]

37. Shpotyuk, O.; Ingram, A.; Shpotyuk, Y.; Filipecki, J.; Shpotyuk, O. Volumetric effects in the degradation of dimethacrylate-based polymer/filler nanocomposites: A positron annihilation study. Polym. Degrad. Stab. 2020, 176, 109150. [CrossRef]

38. Bujňáková, Z.; Kello, M.; Kováč, J.; Tóthová, E.; Shpotyuk, O.; Baláž, P.; Mojžiš, J.; Andrejko, S. Preparation of $\mathrm{As}_{4} \mathrm{~S}_{4} / \mathrm{Fe}_{3} \mathrm{O}_{4}$ nanosuspensions and in-vitro verification of their anticancer activity. Mater. Sci. Eng. 2020, 110, 110683. [CrossRef] [PubMed]

39. Hautojarvi, P.; Pollanen, L.; Vehanen, A.; Yli-Kauppila, J. Vacancies and carbon impurities in $\alpha$-irom: Neutron irradiation. J. Nucl Mater. 1983, 14, 250-259. [CrossRef]

40. Mascher, P.; Dannefaer, S.; Kerr, D. Positron trapping rates and their temperature dependencies in electron-irradiated silicon. Phys. Rev. 1989, 40, 11764-11771. [CrossRef] [PubMed]

41. Liu, M.; Kitai, A.H.; Mascher, P. Point defects and luminescence centers in zinc oxide and zinc oxide doped with manganese J. Lumin. 1992, 54, 35-42. [CrossRef]

42. Vijay, Y.K.; Wate, S.; Awasthi, D.K.; Das, D.; Ghughre, S. Ion induced effects in polymers. Indian J. Eng. Mater. Sci. 2000, 7, 375-377.

43. Shantarovich, V.P.; Goldanskii, V.I. Positron annihilation in free volume elements of polymer structures. Hyperfine Interact. 1998, 116, 67-81. [CrossRef]

44. Wan, Y.; Yang, H.; Zhao, D. "Host-Guest" Chemistry in the Synthesis of Ordered Nonsiliceous Mesoporous Materials. Accounts Chem. Res. 2006, 39, 423-432. [CrossRef]

45. Hill, A.J.; Freeman, B.D.; Jaffe, M.; Merkel, T.C.; Pinnau, I. Tailoring nanospace. J. Molec. Struct. 2005, 739, 173-178. [CrossRef]

46. Dlubek, G.; Taesler, C.; Pompe, G.; Piontek, J.; Petters, K.; Redmann, F.; Krause-Regberg, R. Interdiffusion in a particle matrix system of two miscible polymers: An investigation by positron annihilation lifetime spectroscopy and differential scanning calorimetry. J. Appl. Polym. Sci. 2002, 84, 654-664. [CrossRef]

47. Shpotyuk, O.; Bujňáková, Z.; Baláž, P.; Ingram, A.; Shpotyuk, Y. Positron annihilation lifetime study of polyvinylpyrrolidone for nanoparticle-stabilizing pharmaceuticals. J. Pharm. Biomed. Anal. 2016, 117, 419-425. [CrossRef] [PubMed]

48. Jensen, K.O.; Salmon, P.S.; Penfold, I.T.; Coleman, P.G. Microvoids in chalcogenide glasses studied by positron annihilation. J. Non-Cryst. Solids 1994, 170, 57-64. [CrossRef]

49. Shpotyuk, O.; Filipecki, J. Free Volume in Vitreous Chalcogenide Semiconductors: Possibilities of Positron Annihilation Lifetime Study; WSP: Czestochowa, Poland, 2003; pp. 1-114.

50. Hyla, M.; Filipecki, J.; Shpotyuk, O.; Popescu, M.; Balitska, V. Stoichiometric arsenic sulphoselenides as testing probes for positron trapping in chalcogenide glasses. J. Optoelectron. Adv. Mat. 2007, 9, 3177-3181.

51. Shpotyuk, O.; Golovchak, R.; Ingram, A.; Boyko, V.; Shpotyuk, L. Comparative study of extended free-volume defects in As- and Ge-based glassy semiconductors: Theoretical prediction and experimental probing with PAL technique. Phys. Stat. Sol. 2013, 10, 117-120. [CrossRef]

52. Shpotyuk, O.; Ingram, A.; Shpotyuk, M.; Filipecki, J. Prediction of free-volume-type correlations in glassy chalcogenides from positron lifetime measurements. Nucl. Instr. Meth. Phys. Res. 2014, 338, 66-71. [CrossRef]

53. Shpotyuk, O.; Filipecki, J.; Shpotyuk, M.; Ingram, A. Free volume evolution in chalcogenide glasses as probed by PAL spectroscopy. Solid State Ion. 2014, 267, 38-43. [CrossRef]

54. Ingram, A.; Golovchak, R.; Kostrzewa, M.; Wacke, S.; Shpotyuk, M.; Shpotyuk, O. Compositional dependences of average positron lifetime in binary As-S/Se glasses. Phys. B Condens. Matter 2012, 407, 652-655. [CrossRef]

55. Hyla, M. Compositional anomalies in Se-rich As-Se glass-forming system as revealed from positron annihilation lifetime spectroscopy and ab initio cluster modeling. Comp. Mater. Sci. 2017, 135, 165-168. [CrossRef]

56. Shpotyuk, O.; Hyla, M.; Boyko, V. Compositionally-dependent structural variations in glassy chalcogenides: The case of binary As-Se system. Comp. Mater. Sci. 2015, 110, 144-151. [CrossRef] 
57. Shpotyuk, Y.; Demchenko, P.; Bujňáková, Z.; Baláž, P.; Boussard-Pledel, C.; Bureau, B.; Shpotyuk, O. Effect of high-energy mechanical milling on the medium-range ordering in glassy As-Se. J. Am. Ceram. Soc. 2020, 103, 1631-1646. [CrossRef]

58. Shpotyuk, Y.; Demchenko, P.; Shpotyuk, O.; Balitska, V.; Boussard-Pledel, C.; Bureau, B.; Lukáčová Bujňáková, Z.; Baláž, P. High-energy mechanical milling-driven reamorphization in glassy arsenic monoselenide g-AsSe: On the path tailoring special molecular-network glasses. Materials 2021, 14, 4478. [CrossRef] [PubMed]

59. Shpotyuk, O.; Ingram, A.; Bujňáková, Z.; Baláž, P.; Shpotyuk, Y. Probing sub-atomistic free-volume imperfections in dry-milled nanoarsenicals with PAL spectroscopy. Nanoscale Res. Lett. 2016, 11, 10. [CrossRef] [PubMed]

60. Shpotyuk, O.; Baláž, P.; Bujňáková, Z.; Ingram, A.; Demchenko, P.; Shpotyuk, Y. Mechanochemically driven amorphization of nanostructurized arsenicals, the case of $\beta-\mathrm{As}_{4} \mathrm{~S}_{4}$. J. Mater. Sci. 2018, 53, 13464-13476. [CrossRef]

61. Shpotyuk, O.; Ingram, A.; Baláž, P.; Bujňáková, Z.; Shpotyuk, Y.; Cebulski, J. Free volume studies on mechanochemically-milled $\beta-\mathrm{As}_{4} \mathrm{~S}_{4}$ arsenical employing positron annihilation lifetime spectroscopy. Appl. Nanosci. 2019, 9, 647-656. [CrossRef]

62. Palumbo, G.; Erb, U.; Aust, K.T. Triple line disclination effects on the mechanical behaviour of materials. Scr. Matallurgica Mater. 1990, 24, 2347-2350. [CrossRef]

63. Palumbo, G.; Thorpe, S.J.; Aust, K.T. On the contribution of triple junctions to the structure and properties of nanocrystalline materials. Scr. Matallurgica Materialia. 1990, 24, 1347-1350. [CrossRef]

64. Chattopadhyay, P.P.; Nambissan, P.M.G.; Pabi, S.K.; Manna, I. Polymorphic bcc to fcc transformation of nanocrystalline niobium studied by positron annihilation. Phys. Rev. 2001, 63, 054107. [CrossRef]

65. Lavanya, K.; Senthil, V.; Rathi, V. Pelletization technology: A quick review. Int. J. Pharm. Sci. Res. 2011, 2, 1337-1355. [CrossRef]

66. Hammannavar, P.B.; Lobo, B.; Nambissan, P.M.G. Positron annihilation studies of free volume changes accompanying the incorporation of lead nitrate in PVA-PVP polymeric blend. Rad. Phys. Chem. 2019, 158, 53-60. [CrossRef] 